\title{
Les déséquilibres entre contrat et milieu dans une séance d'histoire à l'école primaire. Une étude exploratoire
}

\section{Didier Cariou}

\section{(Q) OpenEdition}

\section{Journals}

Édition électronique

URL : http://journals.openedition.org/educationdidactique/1422

DOI : 10.4000/educationdidactique. 1422

ISSN : 2111-4838

\section{Éditeur}

Presses universitaires de Rennes

\section{Édition imprimée}

Date de publication : 7 février 2013

Pagination : $9-32$

ISBN : 978-2-7535-2261-9

ISSN : 1956-3485

\section{Référence électronique}

Didier Cariou, « Les déséquilibres entre contrat et milieu dans une séance d'histoire à l'école primaire Une étude exploratoire », Éducation et didactique [En ligne], 7-1 | 2013, mis en ligne le 31 janvier 2015, consulté le 19 avril 2019. URL : http://journals.openedition.org/educationdidactique/1422 ; DOI 10.4000/educationdidactique.1422 


\title{
LES DÉSÉQUILIBRES ENTRE CONTRAT ET MILIEU DANS UNE SÉANCE D'HISTOIRE À L'ÉCOLE PRIMAIRE UNE ÉTUDE EXPLORATOIRE
}

\author{
Didier Cariou \\ IUFM de Bretagne, Université de Bretagne occidentale, CREAD EA 3875
}

Le propos de cet article est de comprendre les raisons de l'inertie du modèle disciplinaire de l'histoire scolaire qui produit, à l'école primaire et dans le secondaire, un enseignement visant surtout la mémorisation et la restitution par les élèves de données factuelles. L'analyse d'une leçon d'histoire au Cours Moyen portant sur l'empire de Charlemagne, considérée comme particulièrement représentative de l'enseignement de l'histoire aujourd'hui, mobilise les outils de la théorie de l'action conjointe en didactique afin de décrire les transactions entre le professeur, les élèves et le savoir historique. Il apparait ainsi que la leçon d'histoire est marquée par une série de déséquilibres entre le contrat didactique et le milieu car le contrat ne permet pas d'exploiter les potentialités du milieu qui parait insuffisamment pourvoyeur des significations nouvelles nécessaires à l'apprentissage. La leçon progresse par une série d'effets de contrat qui obligent le professeur à dévoiler aux élèves le savoir qu'il souhaitait initialement leur faire appréhender par eux-mêmes.

Mots-clés : didactique de l'histoire, modèle disciplinaire, jeux d'apprentissage, conceptualisation historique, théorie de l'action conjointe en didactique.

\section{Lacks of equilibration between contract and milieu in a history lesson at elementary school. An exploratory study}

The aim of this article is to understand the reasons for the inertia of the disciplinary model of scholastic history which simply brings the students, in primary and secondary school, to memorize factual data and to hand them back. The analysis of a history lesson (in fourth elementary school degree) about the Charlemagne's empire, considered as particularly representative of teaching history today, describes the transactions between teacher, students and historical knowledge. In this perspective, we use the model of the Joint Action Theory in Didactics. We focus the point that history lesson is distinguished by several lacks of equilibration between the milieu and the contract because the contract does not allow to develop the potential of the milieu that doesn't seem to provide new meanings for learning. The lesson is going forward through series of effects that bring along the teacher to tell to students the knowledge he initially wanted make them build by themselves.

Keywords: history didactics, scholastic matter model, learning game, historical conceptualization process, joint action theory in didactics. 
Les recherches sur l'enseignement de l'histoire au collège comme à l'école primaire (Audigier, 1995 ; Audigier \& Tutiaux-Guillon, 2004), ont montré la prégnance d'un modèle disciplinaire d'une grande inertie qui s'imposerait à tous les enseignants. L'histoire enseignée serait une discipline " réaliste » qui donnerait directement à voir le réel du passé dans le discours de l'enseignant ou dans les documents utilisés en classe. Ce modèle réaliste gommerait le point de vue qui s'exprime dans chaque document ainsi que les controverses scientifiques qui président à l'élaboration des savoirs historiques. Il en résulte un enseignement factuel visant la mémorisation de faits et de dates, comme l'atteste un rapport de l'inspection générale pour l'école primaire (IGEN, 2005). En effet, l'activité des élèves en classe d'histoire consiste souvent à prélever des informations ponctuelles dans des documents pour répondre aux questions orales de l'enseignant ou à des questions écrites sur des "fiches d'activité » lors de séances de « mise en activité » des élèves. Les démarches formatrices de la conceptualisation, de l'explication et de la périodisation sont le fait de l'enseignant et occupent une place réduite dans la leçon. Ainsi, une recherche a fait apparaitre que les phases explicatives dans le discours du professeur en classe d'histoire au collège occupent moins de deux minutes du total d'une séance. En outre, elles ne sont pas notées par les élèves pour qui prime le contenu factuel (Tutiaux-Guillon \& Mousseau, 1998). Toutefois, aujourd'hui, ce modèle disciplinaire n'est plus adéquat aux attentes d'une société démocratique et aux exigences de formation intellectuelle des futurs citoyens (Audigier, 2005).

Les recherches citées se situent dans la lignée du modèle de la discipline scolaire issu du champ de l'histoire de l'éducation (Chervel, 1998). Une discipline scolaire entretient une grande autonomie par rapport à la discipline universitaire de référence. Elle possède ses propres méthodes d'évaluation, de motivation et de mise au travail des élèves, elle dispense une vulgate largement éloignée des savoirs de référence. Les savoirs de l'histoire scolaire seraient donc simplifiés à l'extrême, emplis de sens commun et parfois même en contradiction avec les avancées récentes de la recherche historique. De fait, l'enseignement de l'histoire repose institutionnellement et historiquement sur une division du travail entre le professeur, qui transmet aux élèves un savoir historique didactisé, et l'historien qui établit scientifiquement les faits à partir d'un problème ou d'une question de recherche qu'il pose aux sources, aux traces du passé (Prost, 1996, p. 56). En effet, selon le mot célèbre de Lucien Febvre: "Pas de problèmes, pas d'histoire ». Poser une question de recherche est la condition d'une « étude scientifiquement conduite » garantissant la validité des assertions de l'historien (Febvre, 1941, p. 22). Comme l'histoire est connaissance par traces, le savoir historique n'est pas donné à l'historien mais construit par une démarche indiciaire (Ginzburg, 1986) et par une argumentation reposant sur l'administration de la preuve documentaire.

La théorie de la transposition didactique (Verret, 1975 ; Chevallard, 1991) rend compte de l'écart entre l'histoire scolaire et l'histoire savante car elle a montré que le savoir scolaire résultait d'une discrétisation du savoir (sa division en objets de savoirs spécialisés) et d'une dépersonnalisation (la séparation du savoir de la personne qui en est à l'origine) qui permettent sa programmabilité (la programmation des apprentissages suivant des séquences successives). Ces contraintes sont les conditions d'une mise en texte et d'un apprêt didactique des savoirs à enseigner. Cette textualisation du savoir conduit à le considérer comme un objet non problématisé, échappant aux exigences de l'argumentation scientifique.

Toutefois, la théorie de la transposition didactique s'oppose au moins sur un point au modèle de la discipline scolaire : elle ne stipule pas l'autonomie du savoir enseigné par rapport au savoir savant puisqu'il en serait une transposition. Ce point précis motive notre démarche qui consistera à se demander quelle est la nature de la relation - rupture ou continuité ? - entre savoir savant et savoir enseigné en classe d'histoire. À cet effet, nous nous livrerons à une étude exploratoire portant sur une séance ordinaire d'histoire au Cours moyen afin de décrire des pratiques effectives de classe. Cette étude devrait nous permettre d'analyser la nature des savoirs et des pratiques de savoir en classe d'histoire. Nous défendons en effet une posture pragmatiste qui envisage les faits didactiques comme des pratiques d'acteurs (le professeur et les élèves) en situation. Nous essaierons d'envisager ce qui conduit à percevoir le savoir historique comme une série d'objets discrétisés à mémoriser et à restituer et ce qui produit en définitive la permanence du modèle disciplinaire de l'histoire enseignée. Nous mobiliserons à cet effet les outils de la théorie des situations didactiques (Brousseau, 1998) et de la théorie 
de l'action conjointe en didactique (Sensevy, 2011) qui permettent de décrire les transactions entre le professeur, les élèves et le savoir historique.

\section{ANALYSER UNE SÉANCE D'HISTOIRE}

\section{Le contexte de la séance analysée}

La leçon de trois séances d'une heure chacune analysée ici a été menée par deux étudiantes de seconde année de Master "métiers de l'enseignement et de la formation » dans le cadre de leur stage pratique en classe de CMl (enfants âgés de 9 ans). La richesse de cette leçon nous conduit à la considérer comme particulièrement exemplaire (Kuhn, 1977 , p. 397) des situations d'enseignement et apprentissage de l'histoire, au sens où elle stabilise un certain nombre de problèmes identifiés par la communauté des didacticiens de l'histoire. Nous considérons également que le recours à la théorie de l'action conjointe en didactique permet de considérer cette leçon comme un cas posant problème (Passeron \& Revel, 2005 ; Doussot, 2012). Il questionne le modèle disciplinaire pour nous donner à voir les raisons de son inertie et nous proposer d'autres possibles. Nous envisageons ainsi l'étude des pratiques de classe objectivées par l'enregistrement vidéo des séances et leur transcription. Pour la clarté du propos, nous avons numéroté les tours de parole (tdp) des trois séances dans la continuité.

La leçon porte sur l'empire de Charlemagne et s'intègre dans le chapitre intitulé dans le programme officiel (BO HS n 3 du 19 juin 2008) : «Après les invasions, la naissance et le développement du royaume de France. "Les repères chronologiques afférant à ce chapitre sont les suivants : « 800 : couronnement de Charlemagne, 987 : Hugues Capet roi de France, Saint-Louis ». Cette série de repères fait apparaitre la naissance du royaume de France - marquée par le couronnement d'Hugues Capet - comme la conséquence du morcellement de l'empire de Charlemagne. La leçon menée par les étudiantes et analysée ici visait l'étude de la nature et du fonctionnement de cet empire autour de l'an 800 .

\section{Préparer une séance d'histoire}

La première difficulté pour un professeur - débutant ou confirmé - qui découvre un programme d'histoire est celle de la nature des savoirs à enseigner aux élèves. Il est en effet impossible de connaitre tous les épisodes de l'histoire de l'humanité, ni même ceux de l'histoire de France. Le professeur doit au préalable s'informer sur la période historique à enseigner, étant entendu que l'acquisition de la connaissance scientifique ne peut s'opérer séparément de la connaissance pédagogique de ce contenu. Cette étape initiale rend compte d'un premier écueil pour l'enseignement de l'histoire.

Lefeuvre (2012) désigne comme une savantisation du savoir le chemin parcouru par l'enseignant pour initier ou approfondir ses connaissances relatives à un savoir donné. Cette démarche lui permet normalement de comprendre le discours des savants du domaine, d'appréhender les problèmes qui ont donné leur forme au savoir et de s'orienter dans un champ du savoir. Il s'agit de s'approprier les propositions considérées comme vraies dans ce champ et de savoir pourquoi elles y sont considérées comme des vérités. En histoire cependant, quand les professeurs acquièrent une connaissance du savoir à enseigner, par manque de temps, ils se confrontent très rarement aux travaux scientifiques de référence et encore moins aux problèmes qui les traversent. Nous trouvons ici l'une des raisons de la permanence du modèle disciplinaire non problématisé de l'histoire scolaire.

Les enseignants se tournent également vers les manuels scolaires du niveau enseigné pour repérer ce qui est essentiel dans ces connaissances et qu'il conviendra de transmettre aux élèves. Ce processus d'essentialisation, qui accompagne selon Lefeuvre (2012) le processus de savantisation, ne désigne pas une simplification du savoir. Il consiste à sélectionner et à stabiliser les éléments jugés les plus importants au regard de ce que l'enseignant estime enseignable aux élèves. L'essentialisation produit ainsi une didactisation du savoir.

Sur le plan épistémique, l'essentiel de la leçon consiste à définir la nature de l'empire de Charlemagne. En nous référant à un ouvrage classique (Riché, 1983), nous en présentons au lecteur les principales caractéristiques qui avaient été transmises aux étudiantes. L'empire peut tout d'abord être défini comme une construction territoriale très vaste 


\section{Didier Cariou}

(environ un million de $\mathrm{km}^{2}$ ) qui englobe des États ou des royaumes en Gaule, en Germanie et en Italie. Ces États furent conquis militairement tout au long du règne de Charlemagne (742-814) dont la titulature (« empereur, roi des Francs et des Lombards») renvoie à cette conception territoriale. Mais le terme d'empire renvoie également à une forme idéologique puisque cette construction politique se réclame de la continuité avec l'empire romain. L'idéologie impériale transparait avec l'épisode du couronnement de Charlemagne à Rome, le 25 décembre 800. La date du couronnement est elle-même symbolique puisque l'on fait ainsi coïncider la naissance du nouvel empire avec celle de Jésus. La cérémonie du couronnement fit se succéder le couronnement et l'adoration du nouvel empereur par le pape puis l'acclamation par la foule et par l'armée. Le modèle est celui de l'empereur Constantin, premier empereur romain qui disait tenir son autorité du Dieu chrétien.

L'empire de Charlemagne est enfin une construction administrative. Dans la lignée des empereurs romains chrétiens, Charlemagne a toute autorité dans les domaines politiques, législatif, judiciaire, militaire et aussi religieux. Il exerce son pouvoir en collaboration avec un conseil restreint et avec l'assemblée des grands dignitaires laïcs, ecclésiastiques et militaires, à l'issue de laquelle étaient adoptées les principales ordonnances. Les décisions prises à la cour d'Aix-la-Chapelle étaient transmises aux comtes représentant l'empereur dans les 300 comtés qui maillaient l'empire. Les comtes avaient en charge l'application des ordonnances impériales, le maintien de l'ordre, la levée des impôts, la justice et la convocation de l'ost des hommes libres. Ces comtes étaient eux-mêmes surveillés par les envoyés de l'empereur, les missi dominici.

Les étudiantes se sont appuyées sur ce savoir essentialisé pour préparer la leçon. La phase de savantisation / essentialisation ayant, selon Lefeuvre (2012), doté l'enseignant d'un potentiel épistémique, celui-ci actualise alors le savoir à enseigner dans une situation concrète d'enseignement apprentissage. Dans la pratique habituelle des enseignants en histoire, cette phase de potentialisation / actualisation du savoir passe par le choix des documents opéré lors de la consultation de manuels scolaires du niveau enseigné. Ces documents sont choisis en fonction de leur « richesse pédagogique », c'est-à-dire en fonction de leur capacité d'actualisation du potentiel épistémique (des savoirs à enseigner). Dans le cas présent, les étudiantes et le chercheur ont supposé que ce potentiel épistémique trouverait à s'actualiser dans l'étude de documents "classiques »: la carte de l'empire de Charlemagne permettrait d'envisager avec les élèves la définition territoriale de l'empire, la photographie de la statue équestre de Charlemagne et une miniature $\mathrm{du} \mathrm{XV}^{\mathrm{e}}$ siècle représentant son sacre, permettraient d'en aborder la définition politique. Enfin, deux textes évoquant les prérogatives des comtes et des missi dominici permettraient d'aborder l'organisation administrative de l'empire. Voici ces documents :

Carte de l'empire de Charlemagne

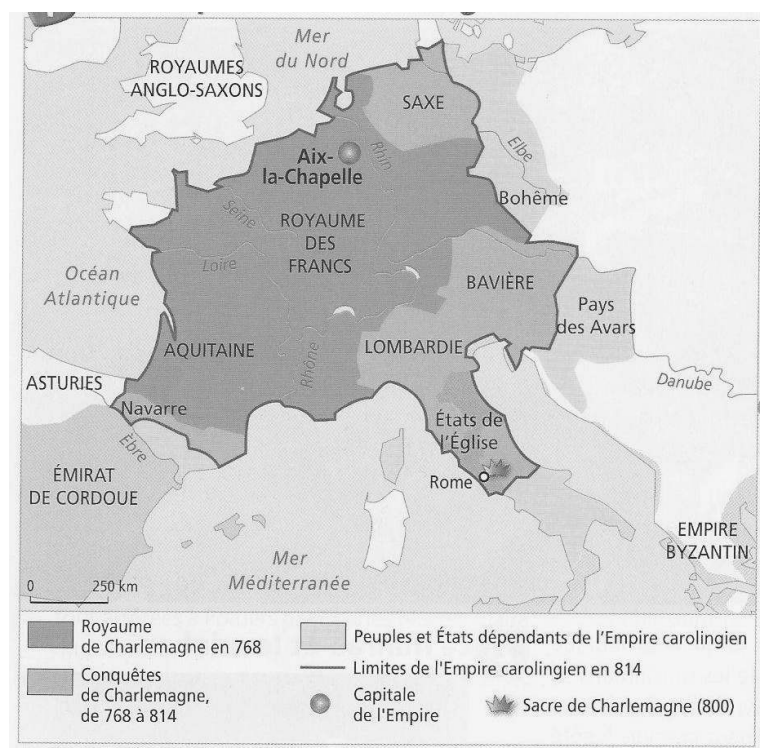

Manuel d'histoire et histoire des arts, classe de CM1, Paris : Nathan, 2010. 
Le couronnement de Charlemagne à Rome par le pape, le 25 décembre 800

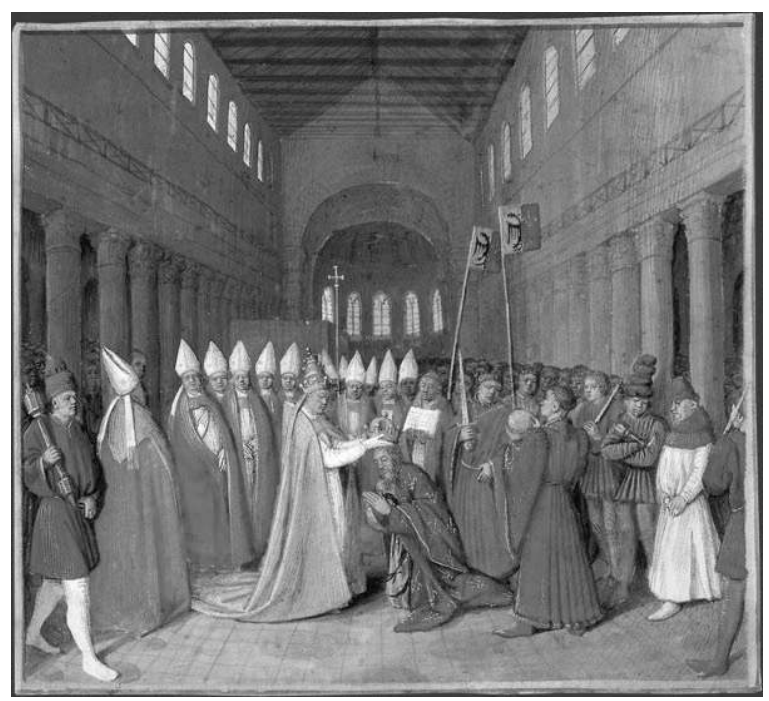

Miniature du $X V^{e}$ siècle. Paris, BnF http://expositions.bnf.fr/carolingiens/grand/fr_6465_089v.htm

Statue équestre de Charlemagne

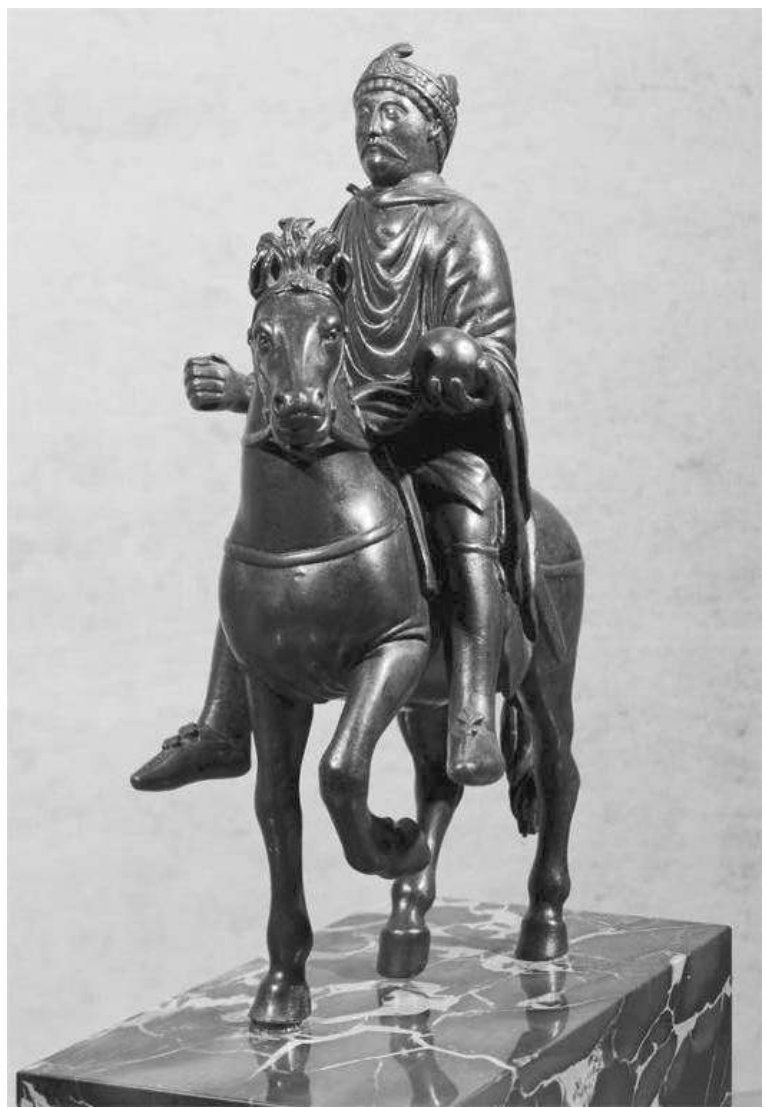

Bronze, IX $X^{e}$ siècle. Musée du Louvre

\section{Texte sur les comtes:}

«Ayant reconnu ta fidélité, je te donne la fonction de comte de cette région pour que tu la diriges et que tu l'administres en me restant toujours fidèle. Tu réprimeras les crimes des bandits et des malfaiteurs. Tu apporteras toi-même à notre Trésor tous les impôts qui lui reviennent. »

D'après un formulaire de désignation de comte, cité par J. Favier, Charlemagne, 1999. Manuel d'histoire et histoire des arts, Classe de CM1, Paris : Nathan, 2010.

\section{Texte sur les Missi Dominici}

«L'empereur Charles a choisi les hommes les plus avisés et les a envoyés dans tout le royaume. Il leur a ordonné de se renseigner très activement et de l'informer sur les pratiques contraires à la loi. Que personne n'ose s'opposer à la loi en vigueur. Que les comtes veillent à ce que les missi puissent aller à travers leur domaine sans être empêchés, s'ils veulent conserver la faveur de l'empereur. »

D'après le Capitulaire des missi dominici, 802. Manuel d'histoire et histoire des arts, Classe de CM1, Paris : Nathan, 2010.

\section{Analyse a priori des séances}

Afin de clarifier les enjeux de l'organisation des séances telles qu'elles avaient été prévues conjointement par le chercheur et les étudiantes, et de repérer les moments de l'étude des trois documents historiques analysés plus loin, nous présentons un tableau qui résume l'analyse a priori de la séance (Sensevy, 2011, p. 151-155).

Le terme d'analyse a priori - ou analyse épistémique - peut s'entendre au sens kantien comme une analyse détachée de l'expérience effective, référée d'une part à ce que l'on sait des savoirs disciplinaires en jeu dans une situation didactique et d'autre part aux configurations possibles de cette situation. L'analyse a priori est un premier outil pour déterminer la nature des savoirs qui constituent l'enjeu des transactions entre le professeur et les élèves, afin de prévoir les potentialités d'action du professeur et les stratégies potentielles des élèves. Elle permet enfin de mettre en évidence les problèmes didac- 


\section{Didier Cariou}

tiques auxquels l'enseignant sera susceptible de faire face. Elle prépare donc l'observation empirique de l'action conjointe du professeur et des élèves autour des savoirs ainsi que la comparaison entre les effets possibles de la situation d'apprentissage et les effets réellement produits

Tableau : Analyse a priori des séances sur l'empire de Charlemagne

\begin{tabular}{|c|c|c|c|}
\hline Analyse des savoirs en jeu & Supports matériels & $\begin{array}{l}\text { Stratégies potentielles des } \\
\text { élèves }\end{array}$ & Difficultés potentielles des élèves \\
\hline \multicolumn{4}{|c|}{ Séance 1 : les définitions de l'empire de Charlemagne } \\
\hline $\begin{array}{l}\text { Définition territoriale: } \\
\text { l'empire domine divers } \\
\text { territoires conquis } \\
\text { militairement }\end{array}$ & Carte de l'empire & $\begin{array}{l}\text { Compétence de lecture de carte. } \\
\text { Repérage de la nature du docu- } \\
\text { ment. } \\
\text { Repérage des espaces représen- } \\
\text { tés. } \\
\text { Repérage des limites de l'empire, } \\
\text { des territoires appartenant à } \\
\text { l'empire, d'Aix-la Chapelle }\end{array}$ & $\begin{array}{l}\text { Reconnaître la nature du document? } \\
\text { Repérer les informations pertinentes? }\end{array}$ \\
\hline $\begin{array}{l}\text { Définition politique : un } \\
\text { ensemble territorial dirigé } \\
\text { par un empereur aux } \\
\text { pouvoirs étendus }\end{array}$ & $\begin{array}{l}\text { Statuette de } \\
\text { Charlemagne }\end{array}$ & $\begin{array}{l}\text { Interprétation de symboles. } \\
\text { Mise en évidence des pouvoirs } \\
\text { de Charlemagne à partir } \\
\text { d'objets symboliques : couronne } \\
\text { (le pouvoir), épée (pouvoir } \\
\text { militaire), sphère (globalité } \\
\text { du pouvoir), croix (pouvoir } \\
\text { d'essence religieuse }\end{array}$ & $\begin{array}{l}\text { Inférer la définition du pouvoir de } \\
\text { Charlemagne à partir d'un objet? }\end{array}$ \\
\hline \multirow[t]{2}{*}{$\begin{array}{l}\text { Définition idéologique : la } \\
\text { légitimation religieuse du } \\
\text { pouvoir impérial }\end{array}$} & $\begin{array}{l}\text { Scène du couronne- } \\
\text { ment de Charlemagne }\end{array}$ & $\begin{array}{l}\text { Lecture d'image. } \\
\text { Repérage du lieu (Rome), de la } \\
\text { date, des personnages (Pape, } \\
\text { Charlemagne), de l'action décrite } \\
\text { Signification politico-religieuse } \\
\text { de la scène }\end{array}$ & $\begin{array}{l}\text { Reconnaître la nature du document? } \\
\text { Inférer la signification de la représen- } \\
\text { tation d'une action? }\end{array}$ \\
\hline & \multicolumn{2}{|c|}{$\begin{array}{l}\text { Production d'un écrit outillé par la liste et définissant } \\
\text { l'empire de Charlemagne }\end{array}$} & \\
\hline \multicolumn{4}{|c|}{ Séance 2 : l'organisation administrative de l'empire de Charlemagne (les comtes) } \\
\hline \multirow[t]{2}{*}{ Les comtes } & $\begin{array}{l}\text { Texte : formulaire } \\
\text { de désignation d'un } \\
\text { comte }\end{array}$ & $\begin{array}{l}\text { Lecture d'un texte historique. } \\
\text { Qui désigne les comtes, quelles } \\
\text { sont leurs attributions et leurs } \\
\text { relations à l'empereur? }\end{array}$ & $\begin{array}{l}\text { Conceptualiser à partir d'une série } \\
\text { d'informations }\end{array}$ \\
\hline & \multicolumn{2}{|c|}{$\begin{array}{l}\text { Production d'un écrit outillé par la liste définissant les } \\
\text { fonctions des comtes et leur relation avec l'empereur }\end{array}$} & \\
\hline \multicolumn{4}{|c|}{ Séance 3 : l'organisation administrative de l'empire de Charlemagne (missi dominici) } \\
\hline \multirow[t]{2}{*}{ Les missi dominici } & $\begin{array}{l}\text { Texte : capitulaire des } \\
\text { missi dominici }\end{array}$ & $\begin{array}{l}\text { Lecture d'un texte historique } \\
\text { Qui désigne les missi, quelles } \\
\text { sont leurs attributions et leur } \\
\text { relation à l'empereur? }\end{array}$ & $\begin{array}{l}\text { Difficulté du vocabulaire } \\
\text { Conceptualiser à partir d'une série } \\
\text { d'informations }\end{array}$ \\
\hline & \multicolumn{2}{|c|}{$\begin{array}{l}\text { Production d'un écrit outillé par la liste définissant } \\
\text { les fonctions des missi dominici et leurs relations avec } \\
\text { l'empereur }\end{array}$} & \\
\hline
\end{tabular}


L'analyse a priori - réalisée ici après coup signale d'emblée les difficultés rencontrées durant cette séance : on perçoit que la nature des documents risque d'obérer les opérations de conceptualisation. Nous émettons donc l'hypothèse que les caractéristiques du modèle disciplinaire, qui apparaîtront à la faveur de l'analyse de cette séance, viennent en partie du choix de ces documents qui nécessiteront des interventions massives du professeur. Le choix de ces documents " classiques »-dans tous les sens du terme - aurait mérité d'être davantage questionné en amont.

\section{Voir une situation didactique comme un jeu}

Afin d'analyser la transcription des transactions entre le professeur et les élèves, nous mobilisons les outils de la théorie de l'action conjointe en didactique qui modélise une situation didactique comme un jeu didactique au cours duquel un professeur fait apprendre un savoir à des élèves (Sensevy, 2011, p. 122-129). Le chercheur regarde ces transactions comme un jeu coopératif où le professeur gagne s'il parvient à faire jouer et à faire gagner ses élèves, c'est-à-dire s'il les conduit à développer d'euxmêmes les stratégies gagnantes qui leur permettront d'apprendre un savoir. L'intérêt de voir une situation didactique comme un jeu - sans affirmer qu'elle est un jeu - est de produire une description des règles définitoires (par exemple au jeu d'échecs, le fou se déplace en diagonale, la tour se déplace en ligne droite, etc.) et des règles stratégiques (par exemple au jeu d'échecs, il est pertinent de placer la tour sur une colonne ouverte) de la situation d'apprentissage (Sensevy, 2011, p. 36-42). Elles permettent alors d'expliquer «à quoi l'on joue » dans un cours d'histoire. Par exemple, sur le plan des règles définitoires $\mathrm{du}$ jeu, les élèves doivent analyser des documents, $\mathrm{y}$ prélever des informations et les confronter afin de répondre aux consignes du professeur. Sur le plan des règles stratégiques du jeu, on considérera que, pour analyser les documents, il est pertinent de faire appel à des savoirs déjà-là, de mobiliser sa mémoire sociale pour tenter de comprendre une situation du passé, d'opérer des comparaisons avec d'autres documents et d'autres situations historiques pour généraliser (Lautier, 1997). Ce type de description envisage également la dynamique des transactions pour expliquer comment le professeur modifie son propre jeu en fonction des réponses et de la stratégie des élèves afin d'infléchir ces dernières pour faire gagner les élèves, c'est-à-dire de les conduire à s'approprier un savoir nouveau. Ainsi, l'action conjointe du professeur et des élèves est modélisée par le chercheur comme un jeu d'apprentissage sous la forme duquel on modélise les pratiques didactiques visant l'acquisition d'un savoir. Le jeu d'apprentissage, que nous cherchons à modéliser à partir d'extraits des séances analysées ici, est celui qui consiste à « faire lire un document historique » (une carte, un texte, un document iconographique).

À un second niveau d'analyse, le chercheur modélise en un jeu épistémique la pratique savante de savoir pour envisager les liens entretenus avec la pratique didactique afin de situer et de décrire cette dernière dans le cadre du jeu d'apprentissage en question. Cette pratique savante est plus précisément modélisée en un jeu épistémique source issu de la modélisation des pratiques expertes du champ scientifique concerné. Ainsi, le jeu d'apprentissage «faire lire un document historique » peut être vu comme un système de trois jeux épistémiques source qui modélisent les pratiques que les historiens nomment la critique externe d'une source (vérification de son authenticité, identification de sa nature, de son auteur, de sa date, de son contexte de production), sa critique interne (analyse du sens littéral des mots employés, détermination du point de vue de l'auteur et des catégories qu'il utilise, croisement de la source avec des sources analogues et avec ce que l'on sait par ailleurs sur le sujet) et enfin la construction, la caractérisation et l'explication des faits historiques à partir de ces sources et à partir d'une question que leur pose l'historien (Prost, 1996, p. 55-77).

En didactique de l'histoire, cette modélisation de la pratique savante des historiens reste encore trop peu construite et nous supposons que la relative méconnaissance des jeux épistémiques sources par la communauté des didacticiens de l'histoire a quelque chose à voir avec l'inertie du modèle disciplinaire de l'histoire enseignée. Nous retiendrons simplement que, dans un fonds d'archives, l'historien mobilise un ensemble de pratiques par lesquelles il entre dans l'archive en s'immergeant dans son étrangeté qui produit un dépaysement par rapport au présent et qui fait fonctionner son imagination. Mais il recourt également à des pratiques qui instaurent une distance à l'archive par sa restitution dans un contexte, par la confrontation à d'autres sources et à d'autres travaux 
d'historiens afin de se représenter la situation des hommes du passé, de tenir compte de leurs savoirs potentiels sur leur situation, et de considérer les différents possibles qui s'offraient à eux, afin de se départir de toute téléologie. L'historien opère ainsi un va-et-vient permanent entre différentes temporalités qui articulent le passé de l'archive au présent de l'analyse (Doussot, 2011, 2012).

En classe, ce jeu épistémique source peut être didactisé en un jeu épistémique émergent qui modélise les pratiques de savoir dont les élèves seraient rendus capables dans leurs transactions avec le professeur et le savoir. L'émergence éventuelle dans l'activité des élèves de comportements qu'on pourrait modéliser comme des jeux épistémiques émergents apparentés aux jeux épistémiques source par lesquels on peut modéliser la pratique savante, concerne non pas des sources primaires mais des documents scolarisés, largement coupés, souvent réécrits et mis en page selon les contraintes des éditions scolaires. Même en tenant compte de cet écart, nous envisageons toutefois la possibilité d'un «nourrissement » des jeux d'apprentissage par les jeux épistémiques source (Sensevy, 2011, p. 741) dont la modélisation s'avère plus que jamais nécessaire.

Les activités didactiques qui nous intéressent ici consistent à " présenter un document» (nature, auteur, date, contexte), à « extraire des informations d'un document " puis à " confronter les informations pour généraliser $»$. Très largement pratiquées dans les classes du secondaire comme du primaire, ces activités résultent d'une modélisation indigène des pratiques savantes des historiens, pensées et formalisées par les rédacteurs des textes officiels pour être appliquées dans les classes. Cette modélisation figure par exemple dans le préambule du programme des classes de seconde (Bulletin officiel spécial $n^{\circ} 4$ du 29 avril 2010) et de première générale (Bulletin officiel spécial $n^{\circ} 9$ du 30 septembre 2010). Cette modélisation scolaire officielle produite par les textes officiels est cohérente avec le mode de conceptualisation spécifique à l'histoire qui procède par " généralisations successives »(Prost, 1996, p. 129). Selon une forme écologique de conceptualisation liée à la spécificité des concepts historiques, la confrontation d'informations différentes sur le même sujet permet, par l'abstraction de leurs différences, d'en dégager leurs caractéristiques communes qui constituent alors les propriétés du concept qui les subsume. On procède de même à partir de concepts concrets de faible degré de généralisation pour construire des concepts plus généraux et plus abstraits. La formulation plus elliptique des programmes de l'école primaire (Bulletin officiel hors-série $\mathrm{n}^{\circ} 3 \mathrm{du} 29$ juin 2008) renvoie également, nous semble-t-il, à cette modélisation scolaire. On invite à développer « chez les élèves curiosité, sens de l'observation et esprit critique $»-$ ce qui englobe selon nous la critique des documents - et le programme d'histoire a pour objectif « d'identifier et de caractériser simplement les grandes périodes de l'histoire » - ce qui recouvre pour nous les opérations de conceptualisation et de périodisation.

Précisons que cette phase de construction du savoir n'est pas évoquée dans le cadre de cet article. Dans la leçon analysée, elle a consisté à lister verticalement sur le tableau les mots désignant les concepts évoqués durant l'analyse des documents (empereur, empire, royaumes, armée, guerres, capitale, pape, couronnement). Après une réflexion collective sur la hiérarchie de ces mots-concepts, les élèves armés de cette liste furent invités à rédiger un texte définissant l'empire de Charlemagne. Le passage de la liste de mots disjoints et structurés verticalement au texte linéaire liant ces mots-concepts (Cariou, 2012, p. 123-129) a favorisé une conceptualisation de l'empire par les élèves.

La modélisation des jeux épistémiques suppose donc que les exercices scolaires ne sont pas totalement des créations scolaires. En ce sens, il semble moins utile d'affirmer l'écart entre les pratiques historiennes et les pratiques de classe, que d'interroger la nature et les modalités de leur transposition afin de comprendre les raisons de l'inertie du modèle disciplinaire de l'histoire scolaire. Il s'agit de se demander à quelles conditions les jeux d'apprentissages, au moyen desquels on peut modéliser et décrire les pratiques didactiques, font émerger des capacités parentes de celles que l'on retrouve dans les jeux épistémiques par lesquels on peut modéliser la pratique des historiens. La description du jeu d'apprentissage montre comment les élèves pourraient s'emparer de ces capacités émergentes que le chercheur modélise comme des jeux épistémiques émergents. Cette description permet également de rendre compte de la progression des transactions entre le professeur et les élèves afin de comprendre la nature des interactions entre le contrat et le milieu en classe d'histoire. 


\section{La mise en jeu d'un contrat et d'un milieu}

La description d'une situation didactique comme un jeu d'apprentissage vise à analyser les transactions entre le professeur et les élèves par lesquelles le professeur conduit ses élèves à mobiliser les stratégies présentes dans le contrat didactique afin que ces derniers interagissent avec un milieu pour s'approprier un savoir nouveau.Le jeu d'apprentissage produit / s'insère en effet dans une dialectique du contrat et du milieu.

Le contrat didactique est un système d'habitudes implicites instituées dans la classe qui engendrent des attentes réciproques entre le professeur et les élèves à propos du savoir et qui permettent aux élèves d'affronter une situation d'apprentissage nouvelle. Ces règles définitoires décrivent le rôle respectif du professeur et des élèves (Brousseau, 1998). On peut aussi les considérer, sous une certaine description, comme les règles définitoires d'un jeu d'apprentissage (Sensevy, 2011, p. 97). Le rôle des élèves est de décoder les intentions du professeur et le rôle de ce dernier est de conduire les élèves à mobiliser certaines stratégies d'apprentissage dans une situation didactique donnée. Toutefois, le contrat habituel en classe d'histoire conduit surtout les élèves à prélever des informations dans des documents.

Le contrat devrait permettre aux élèves de s'orienter dans un milieu que l'on considère comme un ensemble de ressources et de contraintes matérielles et cognitives présentes dans la situation didactique (Brousseau, 1998 ; Sensevy, 2011, p. 111-113). Le milieu est-ce avec quoi l'élève interagit pour apprendre par la mobilisation des règles stratégiques $\mathrm{du}$ jeu. Il actualise un ensemble de règles, de savoir déjà acquis, d'objet matériel mais aussi de savoirs nouveaux à acquérir. Selon les deux acceptions courantes du mot, le milieu constitue l'environnement du jeu d'apprentissage (en classe d'histoire, on étudie des documents afin de répondre aux questions du professeur), mais il est aussi ce qui fait obstacle et qui se trouve entre le professeur et les élèves (en classe d'histoire, on cherche à comprendre et interpréter ces documents). En fonction des « rétroactions » du milieu, les élèves doivent mobiliser des règles stratégiques rendues disponibles par le contrat didactique telles que la mobilisation des savoirs historiques déjà acquis, des méthodes de lecture et de critique des documents. Le milieu est donc, d'une certaine façon, nécessairement " antagoniste au contrat présent », il doit résister et provoquer un déséquilibre avec ce contrat car, s'il suffisait d'appliquer tel quel le contrat dans toute situation didactique nouvelle, alors les élèves n'apprendraient rien de nouveau. De même, si le milieu n'est pas perçu comme antagoniste au contrat (par exemple, si l'on considère qu'il suffit d'une lecture littérale du document pour le comprendre et l'interpréter) alors les élèves ne se confrontent pas non plus au milieu. La rupture de l'équilibre dans une activité donnée et le problème qui en découle suscitent le besoin de la restauration de l'équilibre à la faveur d'une enquête (Dewey, 1938, p. 84-85). La réussite de l'activité didactique, modélisée en terme de « gain » au jeu d'apprentissage dépendra du rétablissement de l'équilibre entre un milieu antagoniste et un contrat didactique renouvelé qui aura intégré des démarches et des savoirs nouveaux par les interactions avec ce milieu. Cette équilibration didactique nouvelle signale l'effectivité de l'apprentissage et fait avancer le temps didactique.

Nous allons donc observer la question de l'équilibre entre contrat et milieu lors d'une leçon d'histoire en suivant l'ordre du quadruplet des règles définitoires du jeu (définition, dévolution, régulation et institutionnalisation) (Sensevy, 2011, p. 143-146) pour décrire la progression du jeu d'apprentissage « jouer à lire des documents historiques » à savoir : la carte de l'empire de Charlemagne, une miniature du $\mathrm{XV}^{\mathrm{e}}$ siècle représentant le couronnement de Charlemagne et le texte sur les comtes.

\section{LA DÉFINITION DE LA SITUATION}

La première étape du jeu d'apprentissage consiste en une définition de la situation d'apprentissage. En histoire, cette étape est celle de la « présentation du document $»$. On peut modéliser cette étape comme un jeu épistémique émergent apparenté à la pratique savante elle-même modélisée comme le jeu épistémique source de la critique externe du document. Pour chacun des trois documents étudiés, nous observons que les termes du contrat présent dans la classe (deviner les intentions du professeur, rechercher des informations dans les documents) ne permettent pas de reconnaitre les spécificités et les potentialités du milieu. 


\section{Didier Cariou}

\section{Reconnaître une carte historique}

Le premier document étudié lors de la leçon est la carte de l'empire de Charlemagne.

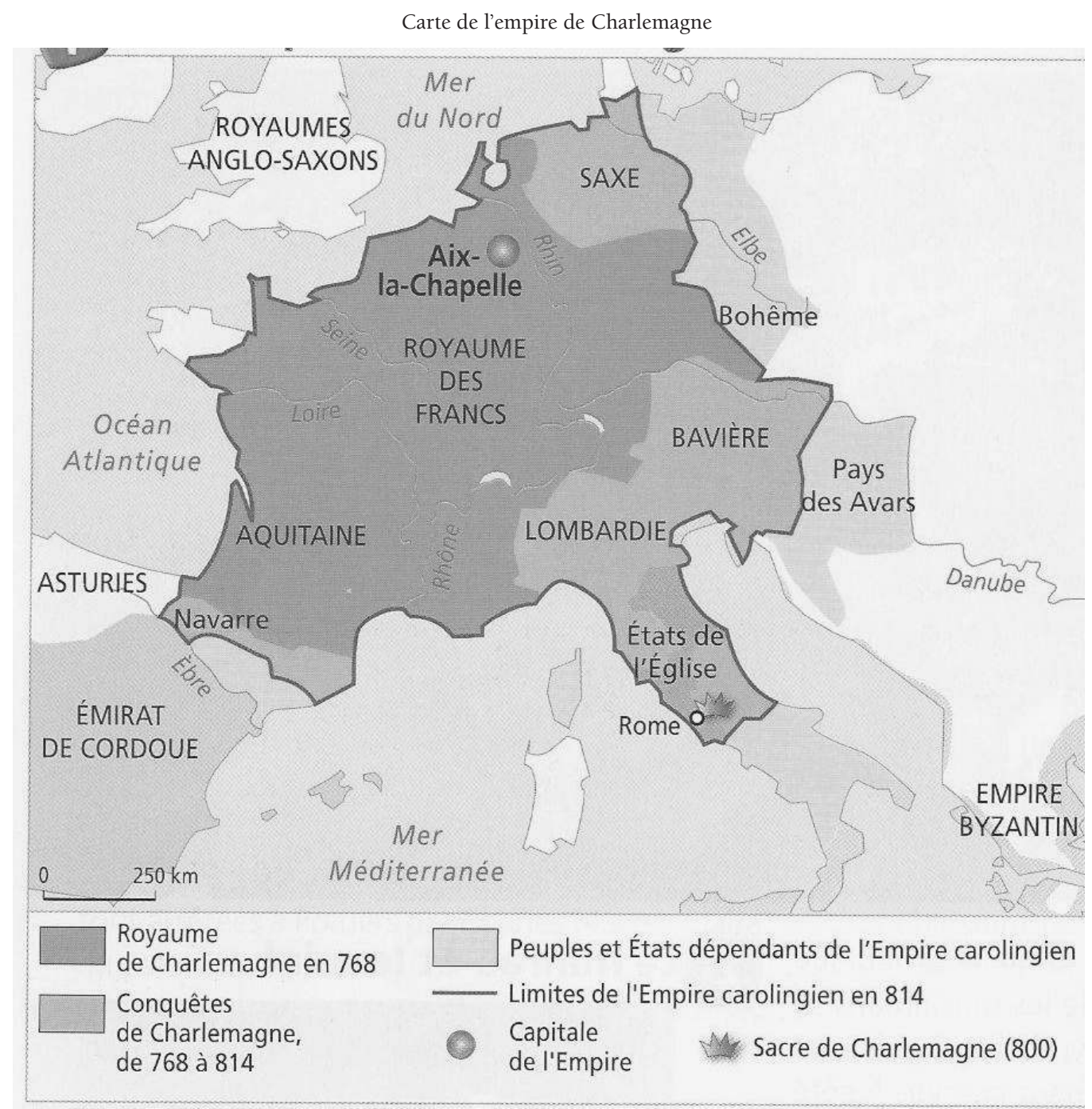

Manuel d'histoire et histoire des arts, classe de CM1, Paris : Nathan, 2010.

L'extrait suivant pointe la difficulté à reconnaitre la nature de ce document.

\begin{tabular}{|l|l|l|}
\hline 11 & Prof & Est-ce que vous pouvez me dire ce que c'est que ce document ? Guillaume ? \\
\hline 12 & Guillaume & C'est l'empire de Charlemagne. \\
\hline 13 & Prof & D'accord. Oui, c'est le titre, "L'empire de Charlemagne". Et quel type de document c'est? Alexandre? \\
\hline 14 & Alexandre & Euh, c'est tous les pays qu'il a conquis. \\
\hline 15 & Prof & $\begin{array}{l}\text { Qu'il a conquis. Donc ce sont des territoires qu'il a conquis avec son armée. Mais l'ensemble, c'est } \\
\text { un document, c'est une carte. D'accord ? }\end{array}$ \\
\hline
\end{tabular}


Ces échanges sont significatifs des réponses généralement apportées à ce type de question qui inaugure toute lecture de document en classe d'histoire, dans le primaire comme dans le secondaire. Les élèves ne parviennent pas à déterminer la nature de ce document car ce dernier est identifié selon son titre figurant dans la légende du document (tdp 12 : « C'est l'empire de Charlemagne ») ou selon les informations qu'il peut fournir (tdp 14: «C'est tous les pays qu'il a conquis $»$ ). Un effet du contrat habituel en classe d'histoire est que les documents n'existent pas en eux-mêmes, ils sont transparents à la réalité et leur nature importe moins que les informations qu'ils peuvent fournir sur le passé (Tutiaux-Guillon, 2009). Nous retrouvons ici la dimension « réaliste» du modèle disciplinaire.

Conformément aux attendus du modèle disciplinaire dominant, cette présentation du document s'opère en préalable à l'étude du document lui-même. Elle ne permet pas aux élèves de le reconnaitre, de le lire, d'en explorer le système symbolique, de se confronter aux potentialités du milieu pour produire du sens à partir de ce document. Il s'agissait pourtant de reconnaitre que ce document est une carte historique représentant un ensemble politique constitué aux alentours de l'an 800, que cet empire regroupe de nombreux royaumes et territoires (définition territoriale de l'empire) et qu'il est engagé dans une dynamique d'expansion territoriale (explication de la taille de cet empire). Cependant, les élèves cherchent seulement à deviner les intentions du professeur et à mobiliser des stratégies contractuelles présentes (chercher des informations dans un document) mais inadéquates au milieu nouveau car elles ne leur permettent pas d'envisager la spécificité du milieu nouveau (ce document est une carte et pas l'empire de Charlemagne). On considère alors que le contrat « envahit » le milieu et empêche les élèves de se confronter aux potentialités offertes par ce dernier. Quand il s'avère ainsi que le contrat n'est pas « adjuvant $»$ au milieu, la restauration de l'équilibre entre le contrat et le milieu s'avère irréalisable (Sensevy, 2011, p. 194-196 et p. 255-259).

Cet envahissement du milieu par le contrat produit un glissement du jeu d'apprentissage (Marlot et Théry, 2011) car, pour faire progresser le temps didactique, le professeur est obligé de délivrer aux élèves la réponse attendue (tdp 15 : « c'est une carte »). En effet, si les élèves savent ce qu'est une carte, le contrat présent dans la classe les empêche de comprendre que la question posée suppose une identification du document comme une carte historique. Ce glissement du jeu produit des effets de contrat. Lors de la phase de construction du savoir par les élèves, le professeur se voit contraint de suggérer fortement la réponse aux élèves et leur permet de répondre à la question sans savoir. C'est une forme d'effet Topaze auquel s'apparente la réponse de Guillaume (tdp 12) qui identifie la nature du document par la simple lecture de la légende du document. Lors de la phase d'évaluation du savoir produit, le professeur affecte de reconnaitre dans la réponse des élèves les indices d'un savoir qui ne s'y trouve pas en réalité. C'est une forme d'effet Jourdain auquel s'apparente l'identification par le professeur du document comme une carte (tdp 15) (Brousseau, 1998, p. 52-53 ; Sensevy, 2011, p. 78-79 et p. 281-286).

Lors de la seconde séance et du retour sur cette carte, la difficulté à reconnaitre la nature de ce document apparait à nouveau.

\begin{tabular}{|l|l|l|}
\hline 385 & Prof & Alors, comment t'as su que c'était l'empire de Charlemagne ? \\
\hline 386 & Guillaume & Ben, y a les documents \\
\hline 387 & Prof & Alors, moi, si je n'avais pas de documents, comment tu m'expliquerais ? \\
\hline 388 & Guillaume & Ben, ça parle de Charlemagne \\
\hline 389 & Prof & Qu'est-ce qui parle de Charlemagne? \\
\hline 390 & Guillaume & Ben, la fiche. \\
\hline 391 & Prof & La fiche ? Est-ce qu'on peut être plus précis ? \\
\hline 392 & Élève & Le document. \\
\hline 393 & Prof & Le premier document, c'est quoi ? \\
\hline 394 & Guillaume & Hum, c'est l'empire. \\
\hline 395 & Prof & C'est quoi comme document ? Une... \\
\hline 396 & Alexandre & Une carte \\
\hline 397 & Guillaume & Une carte \\
\hline 398 & Prof & Une carte \\
\hline
\end{tabular}




\section{Didier Cariou}

Les échanges entre le professeur et Guillaume montrent l'impossibilité pour ce dernier à nommer la carte, à en dire la nature et la spécificité. En histoire, les documents seraient tous identiques et transparents à leur objet puisque Guillaume assimile la carte aux « documents » puis à la « fiche » et ces derniers ne vaudraient que parce qu'ils fourniraient des informations sur « l'empire ». La nécessité pour le professeur de faire identifier malgré tout la carte par les élèves produit alors un effet Topaze (tdp 395 et 396 : «C'est quoi comme document ? Une...»/ «Une carte »). Les caractéristiques de ces transactions orales montrent un déséquilibre dans la topogenèse, c'est-à-dire dans les places occupées respectivement par le professeur et les élèves lors de cette situation d'apprentissage. Le professeur, en position haute, maitrise la situation et guide les réponses des élèves y compris par des effets de contrat. Les élèves, de leur côté, se trouvent en position basse, ils en sont réduits à deviner les intentions du professeur. Ils ne sont pas pourvus du langage pour parler en histoire et pour dire qu'ils ont une carte sous les yeux. La désignation des objets du milieu fait défaut.

Cette première analyse suggère que la permanence et l'inertie du modèle disciplinaire tient peutêtre à ce type d'interactions qui ne présente que les apparences du dialogue.

\section{Déterminer la nature d'un document iconographique}

Le couronnement de Charlemagne à Rome par le pape, le 25 décembre 800.

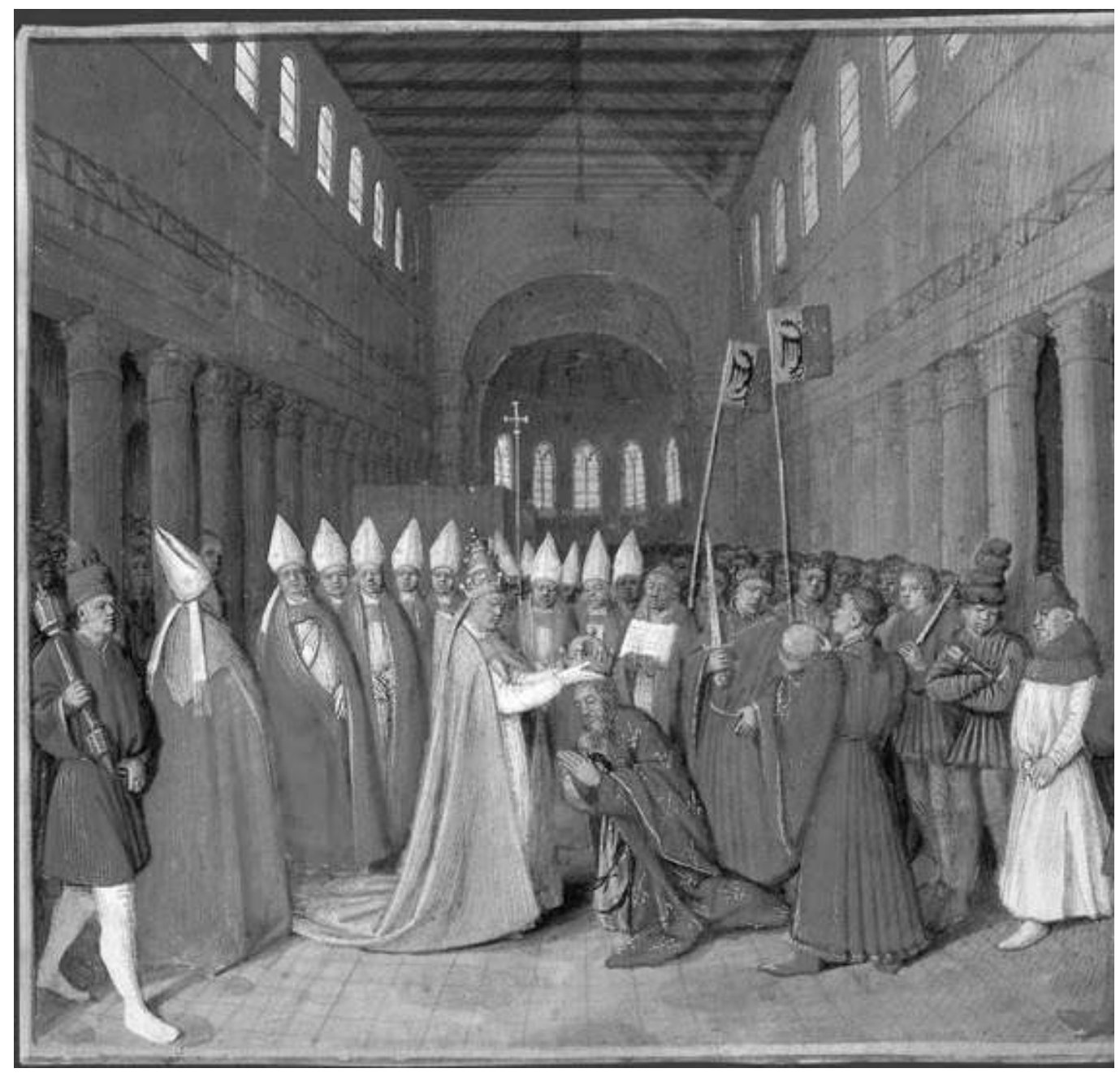

Miniature du $X V^{e}$ siècle, Paris, Bibliothèque nationale de France 
L'appréhension réaliste des documents parait encore plus nettement à l'occasion de la présentation de la miniature du $\mathrm{XV}^{\mathrm{e}}$ siècle représentant le couronnement de Charlemagne à Rome en l'an 800. En se fiant à la légende du document, les élèves déterminent la nature du document ainsi que la date et le lieu de l'action représentée, sans vraiment regarder le document. C'est alors qu'intervient l'échange suivant : basilique romaine et pas la cathédrale de Reims. Le document offre la représentation par les hommes $\mathrm{du} \mathrm{XV} \mathrm{V}^{\mathrm{e}}$ siècle d'un événement qui s'était produit en 800. Toutefois, en raison des habitudes induites par le contrat, un jeu épistémique parent de la critique externe du document, qui pourrait émerger de la présentation du document, s'avère difficile à jouer ici.

\begin{tabular}{|l|l|l|}
\hline 153 & Prof & Tu voulais rajouter quelque chose Mia? \\
\hline 154 & Mia & Et c'est une vraie image ? \\
\hline 155 & Prof & $\begin{array}{l}\text { C'est un tableau, ça a été peint. C'est pas de l'époque, parce que vous avez vu que c'était au XV } X^{e} \text { siècle, } \\
\text { c'est une miniature qui date du XV } \\
\text { l'an } 800 . \text { On nècle sauf que Charlemagne, il s'est fait sacrer empereur en la même date. Là on est en } 1400 \text { et des brouettes dans le tableau, et ici on } \\
\text { est en } 800 .\end{array}$ \\
\hline
\end{tabular}

La remarque de Mia met en évidence une nouvelle contradiction entre le contrat et le milieu. Si le contrat didactique suppose la transparence des documents au réel du passé - puisqu'ils nous fournissent des informations sur le passé - alors cette image devrait nécessairement être «vraie » ou réaliste et avoir été produite au moment même où s'est déroulée la scène représentée. Or, le document proposé fut réalisé 600 ans plus tard et il représente les principaux protagonistes dans des costumes et des attitudes du $\mathrm{XV}^{\mathrm{e}}$ siècle. La remarque de Mia pointe l'aporie du contrat didactique habituel en classe d'histoire. Le repérage de la date et du lieu de l'action, ainsi que des personnages représentés conduit à lire les documents comme s'ils étaient « vrais ", comme s'ils donnaient directement à voir l'action passée elle-même,

La réponse du professeur (tdp 155) signale la nécessité d'une critique interne exhaustive du document par laquelle les élèves se confronteraient au milieu et prendraient conscience de l'écart temporel entre la scène représentée et sa représentation. La pratique des historiens consiste plutôt à confronter cette image aux miniatures $d u \mathrm{XV}^{e}$ siècle qui représentent des couronnements royaux contemporains. Il en ressortirait alors que ce qui nous est donné comme le couronnement de Charlemagne est plutôt représenté comme le couronnement d'un monarque capétien : présence des prélats et des Grands du royaume, costume de sacre, présentations des insignes du pouvoir royal (couronne, épée, sceptre, main de justice, etc.). Trois éléments seulement évoquent une autre période : les deux personnages centraux sont le pape et l'empereur et non pas l'archevêque de Reims et un capétien, le lieu est une

\section{Se repérer dans un texte}

Une autre opération induite par la présentation du document consiste à s'assurer de la compréhension littérale du texte avant d'en proposer une interprétation. Cette coutume didactique dans les classes d'histoire découle d'une contrainte anthropologique propre aux textes historiques (Sensevy \& Rivenc, 2003, p. 75-76 ; Koselleck, 1997, p. 230). En effet, le texte et le réel passé de l'histoire, le discours présent et l'action passée ne se superposent jamais parfaitement. Les textes anciens décrivent le passé au prisme des catégories de leurs auteurs. Leur discours relève donc d'un point de vue qui suppose la mise à distance par la critique interne et externe de la source. Dans la modélisation de la pratique historienne comme un jeu épistémique source, l'une des règles définitoires du jeu consiste à bannir toute naïveté face aux mots rencontrés dans une source et à s'interroger sur leur sens à l'époque de la source. Par exemple, dans les sources médiévales, le mot latin servus désigne-t-il un esclave - au sens antique du terme - ou bien un serf ? Une recherche s'avère alors nécessaire pour établir à quel moment dans les campagnes de l'occident médiéval, derrière le mot servus, le serf a succédé à l'esclave (Bloch, 1949, p. 137). Dans le passage suivant, un décrochage s'opère donc entre le jeu épistémique source, que nous venons d'esquisser très grossièrement, et le jeu d'apprentissage. En effet, le texte utilisé est traduit en français et réécrit dans un langage supposé accessible aux élèves. En outre, le professeur veut s'assurer de la compréhension littérale par les élèves de certains mots du texte quand ces derniers supposeraient une 
définition complexe (en gras dans le texte que nous reproduisons à nouveau ici) :

« Ayant reconnu ta fidélité, je te donne la fonction de comte de cette région pour que tu la diriges et que tu l'administres en me restant toujours fidèle. Tu réprimeras les crimes des bandits et des malfaiteurs. Tu apporteras toi-même à notre Trésor tous les impôts qui lui reviennent. »

Nous présentons un court extrait des échanges sur le vocabulaire du texte. jeu, afin que les élèves produisent cette stratégie de leur propre mouvement et fournissent ainsi la preuve de ce qu'ils ont appris quelque chose (Sensevy, 2011, p. 69-70). De fait, le professeur articule la réticence (« je ne vais pas vous dire ce que c'est un comte») à l'expression qui vise à provoquer l'action de l'élève en vertu de la valeur perlocutoire de l'énoncé (« sinon j'aurais fait le travail à votre place ", i.e. : dites-moi ce qu'est un comte). Le couple réticence / expression rappelle que, tout en retenant de l'information, le professeur doit cependant exprimer quelque chose pour provoquer l'action des élèves (Sensevy, 2011,

\begin{tabular}{|l|l|l|}
\hline 499 & Prof & $\begin{array}{l}\text { Est-ce qu'il y a des mots que vous n'avez pas compris ? que ne vous permettent pas de comprendre } \\
\text { le texte? }\end{array}$ \\
\hline 500 & Marie & Comte \\
\hline 501 & Prof & $\begin{array}{l}\text { Alors, justement, comte, ce petit texte va permettre d'écrire une liste sur les comtes. Donc je ne vais } \\
\text { pas encore vous dire ce que c'est un comte sinon j'aurais fait votre travail à votre place. Alors, celui-là } \\
\text { on le laisse de côté, on verra à la fin ce que c'est un comte. Chloé ? }\end{array}$ \\
\hline 502 & Chloé & Malfaiteur \\
\hline 503 & Prof & Malfaiteur \\
\hline 504 & Élèves & Des bandits, c'est pareil que bandits. \\
\hline 505 & Prof & C'est pareil que bandits. C'est des gens qui font le mal. Alors, quoi d'autre? \\
\hline
\end{tabular}

L'explicitation du sens de «malfaiteur» en « bandit » montre que l'on vise une lecture littérale du texte qui ne suppose aucune interprétation de la part du lecteur. Cette démarche produit encore la persistance du modèle disciplinaire et sa logique positiviste d'appréhension du texte comme s'il suffisait de « traduire » les mots du texte pour accéder au réel du passé.

D'autre part, l'échange avec Marie (tdp 500 et 501) montre que les élèves attendent du professeur qu'il définisse la signification de tous les mots du texte, y compris le mot « comte ». Le contrat apparait à nouveau comme envahissant le milieu dans le sens où les élèves s'en tiennent au contrat présent (produire une lecture littérale du texte) quand le professeur vise le renouvellement du contrat par la confrontation à ce milieu nouveau (construire le concept de comte à partir des informations fournies par le texte). On perçoit cette contradiction dans la réticence du professeur à fournir cette définition (tdp 501 : « Je ne vais pas encore vous dire ce que c'est un comte sinon j'aurais fait votre travail à votre place »). Par la réticence, le professeur se retient de divulguer tout ce qu'il sait à propos de la stratégie gagnante au p. 390-394 et p. 483). Dans les échanges analysés ici, l'équilibre entre réticence et expression parait fragilisé car le contrat et les faibles potentialités du milieu poussent du côté de l'expression. La contradiction apparait encore au moment où le professeur veut s'assurer que les élèves ont identifié les interlocuteurs du texte, à savoir Charlemagne et le comte.

Eva (tdp 525) identifie l'auteur du texte comme étant le pape. Dans le document iconographique étudié précédemment, le pape était effectivement l'auteur de l'action du couronnement de Charlemagne. L'élève mobilise donc une connaissance intégrée au contrat didactique mais inadéquate au nouveau milieu. En outre, cette remarque signale que l'un des termes du contrat présent dans la classe est la reconnaissance d'un savoir déjà institutionnalisé dans la classe, ce qui produit l'envahissement du contrat par le milieu. Cette caractéristique du contrat stipule que le savoir ne se construit pas mais se reconnait ou se devine.

L'autre difficulté signalée par cet extrait tient à l'absence de reconnaissance du comte comme destinataire du texte rédigé au nom de Charlemagne. Cette fois, le milieu est totalement antagoniste 


\begin{tabular}{|l|l|l|}
\hline 524 & Prof & $\begin{array}{l}\text { Qui est le "je" dans le texte ? À la première ligne, il y a un « je ». C'est qui ce « je », qui est-ce qui } \\
\text { parle ? On lève le doigt. Eva? }\end{array}$ \\
\hline 525 & Eva & Le pape \\
\hline 526 & Prof & Ah ! Est-ce que c'est le pape? \\
\hline 527 & Élèves & Non \\
\hline 528 & Prof & Non, ce n'est pas le pape. Elouan ? \\
\hline 529 & Elouan & Charlemagne \\
\hline 530 & Prof & C'est Charlemagne. C'est Charlemagne qui parle. Et, il parle à qui ? \\
\hline$[\ldots]$ & & \\
\hline 534 & Elouan & Un soldat \\
\hline 535 & Prof & $\begin{array}{l}\text { Non, ce n'est pas un soldat non plus. Alors, je vous aide, on verra pourquoi il s'adresse à eux. Il s'agit } \\
\text { des comtes en fait. Il parle aux comtes. Alors, si on sait ça, si on sait que, que Charlemagne parle } \\
\text { aux comtes [...] }\end{array}$ \\
\hline
\end{tabular}

au contrat : l'intention du professeur est de faire construire par les élèves le concept de comte à partir des informations fournies par le texte - d'où sa réticence manifestée au tdp 501 - en conformité avec le jeu épistémique qui modélise la construction des faits historiques. Mais, dépourvus du concept de comte, les élèves ne peuvent ni identifier le destinataire du texte ni comprendre ce texte. Il est donc nécessaire au professeur de relâcher sa réticence et de délivrer aux élèves l'identité du destinataire (tdp 535). Ce faisant, le professeur oriente l'action des élèves du côté du contrat, du côté de la stratégie de la reconnaissance des intentions du professeur et de la devinette. La relaxation de la réticence (Sensevy, 2011, p. 245) produit / signale un affaiblissement des potentialités du milieu.

Les caractéristiques du contrat comme celles du milieu produisent alors un glissement du jeu d'apprentissage, elles empêchent la réticence comme la dévolution du jeu.

\section{L'IMPOSSIBLE DÉVOLUTION ET L'IMPOSSIBLE RÉGULATION DU JEU DIDACTIQUE}

\section{Les phases de dévolution, de régulation et d'institutionnalisation}

La seconde phase du quadruplet des règles définitoires du jeu d'apprentissage est celle de la dévolution (Brousseau, 1998, p. 301-303 ; Sensevy, 2011, p. 67-75). Exprimé théoriquement, on peut dire que le professeur s'efforce de dévoluer le jeu aux élèves et de leur en confier - au moins en partie - la responsabilité. Il veut s'assurer ainsi que les élèves produiront un rapport adéquat au milieu pour mettre d'euxmêmes en œuvre une stratégie qui les conduise sur le chemin du savoir à construire, comme l'échange ci-dessus vient de le montrer a contrario. La dévolution suppose en effet, du côté du professeur, une forme de réticence à dévoiler tout ce qu'il sait, afin que les élèves « jouent le jeu », et déploient d'euxmêmes, proprio motu, les stratégies d'apprentissage pertinentes. Si le professeur dévoilait aux élèves les connaissances nécessaires au déploiement de la stratégie gagnante, alors les élèves ne pourraient pas produire cette stratégie de leur propre chef et il n'est pas certain qu'ils puissent apprendre quelque chose. Par la clause proprio motu, l'élève accepte d'abandonner le déchiffrement des intentions du professeur pour se confronter aux choses du monde actualisées dans un milieu nouveau et que le contrat présent ne peut pas totalement assimiler (Sensevy, 2011, p. 199). Toutes proportions gardées, nous retrouvons ici la première phase du modèle intermédiaire d'apprentissage de l'histoire à l'occasion de laquelle, face à une situation historique pas encore connue, les élèves mobilisent d'eux-mêmes des savoirs scolaires déjà-là et des éléments de leur mémoire sociale pour proposer des hypothèses interprétatives qui permettront l'assimilation d'un savoir historique nouveau (Lautier, 1997). Pourtant, dans ces séances d'histoire où le contrat envahit le milieu, la dévolution s'avère malaisée et elle est enchâssée dans des phases de régulation du jeu

Au cours du déroulement du quadruplet du jeu, la phase de régulation conduit en effet le professeur à réguler le comportement des élèves afin qu'ils produisent les stratégies gagnantes en leur rappelant les règles du jeu, en infléchissant leur stratégie, en 
validant ou en invalidant leurs réponses. Cette régulation est orientée vers l'équilibration du contrat et du milieu. Cette phase de régulation est suivie d'une phase d'institutionnalisation qui reconnait la validité du savoir construit par les élèves à l'occasion de ce jeu d'apprentissage. Dans la séance, l'institutionnalisation passe par l'inscription au tableau des mots relevés lors de l'étude des documents. Ce savoir nouveau est institutionnalisé dans la classe comme un savoir partagé dans l'arrière-plan du contrat didactique spécifique à la classe (Brousseau, 1998 ; Sensevy, 2011, p. 145-146).

Du point de vue de la didactique de l'histoire, il nous semble important de rappeler que ces phases de régulation et d'institutionnalisation correspondent à la seconde phase du modèle d'apprentissage de l'histoire durant laquelle le professeur conduit les élèves à mettre à distance leur interprétation de la situation historique étudiée afin de la vérifier, de la valider et de la formaliser à des fins d'institutionnalisation (Lautier, 1997). Il nous semble donc possible d'envisager une convergence entre ce modèle essentiel de la didactique de l'histoire et les modèles élaborés à l'origine dans le cadre de la didactique des mathématiques. Un examen plus approfondi de cette convergence serait souhaitable pour nourrir une didactique comparée.

Nous allons tenter de comprendre comment opèrent ces deux phases du jeu d'apprentissage dans la séance.

\section{Interpréter une scène historique}

Revenons à la miniature qui représente le couronnement de Charlemagne par le pape en l'an 800. Une fois repérés la nature du document, la date et le lieu de l'action ainsi que les personnages représentés (critique externe du document), il convient maintenant d'en proposer une interprétation (critique interne) pour construire le savoir historique. Dans le passage ci-dessous, le professeur tente de dévoluer aux élèves la responsabilité de l'interprétation de la scène représentée (tdp 169 : « Il devient empereur et, du coup, qu'est-ce qu'il peut obtenir comme pouvoir?»).

\begin{tabular}{|c|c|c|}
\hline 167 & Prof & $\begin{array}{l}\text { Donc il couronne Charlemagne. Donc c'est une couronne, c'est le pape qui pose la couronne sur la } \\
\text { tête de Charlemagne. À votre avis, pourquoi il pose cette couronne sur la tête de Charlemagne? } \\
\text { Marie? }\end{array}$ \\
\hline 168 & Marie & Parce qu'il devient l'empereur. \\
\hline 169 & Prof & Il devient empereur et, du coup, qu'est-ce qu'il peut obtenir comme pouvoir? Vincent? \\
\hline 170 & Vincent & Il dirige le peuple chrétien \\
\hline 171 & Prof & Oui, d'accord. Donc il reçoit son pouvoir de qui ? Cassandra? \\
\hline 172 & Cassandra & Du pape. \\
\hline 173 & Prof & Et au-dessus du pape, il y aurait qui ? Oui? \\
\hline 174 & Elouan & L'oiseau... \\
\hline 175 & Prof & Ah, tu confonds avec Clovis, la colombe qui amène... \\
\hline 176 & Elouan & Oui, l'ampoule. \\
\hline 177 & Prof & $\begin{array}{l}\text { L'ampoule, oui. Alors, au-dessus du pape en fait le pape est représentant de Dieu donc Charlemagne, } \\
\text { en se faisant sacrer par le pape, écoutez bien, en se faisant sacrer par le pape, Charlemagne reçoit les } \\
\text { pouvoirs de Dieu. Est-ce que tout le monde a compris? }\end{array}$ \\
\hline 178 & Élèves & Oui \\
\hline 179 & Prof & $\begin{array}{l}\text { Est-ce que tout le monde a compris? Est-ce que quelqu'un peut me redire ce que je viens d'expli- } \\
\text { quer, me le redire avec ses mots? Tissia, tu peux me redire avec tes mots pourquoi c'est le pape qui } \\
\text { couronne Charlemagne? }\end{array}$ \\
\hline 180 & Tissia & Parce que... \\
\hline 181 & Prof & Doriane, tu peux l'aider ? \\
\hline 182 & Doriane & Parce que... \\
\hline 183 & Prof & Alors, qui peut aider? Vas-y Vincent, on t'écoute \\
\hline 184 & Vincent & $\begin{array}{l}\text { En fait le pape il est, heu, le pape il est heu, au-dessus du pape il y a Dieu et quand il se fait couron- } \\
\text { ner, il obtient les pouvoirs de Dieu comme heu... }\end{array}$ \\
\hline 185 & Prof & Voilà \\
\hline 186 & Vincent & Comme celui qui est entre Dieu et le pape \\
\hline
\end{tabular}


Dans cet extrait, la dévolution suppose un certain nombre de régulations de la part du professeur situées à la limite de l'effet Topaze (tdp 171 : «Il reçoit son pouvoir de qui ?»; tdp 173 : «Au-dessus du pape il y aurait qui ?»). Ces dernières visent la production par les élèves d'une hypothèse explicative à partir d'une interprétation symbolique du document. C'est du moins ainsi que nous pourrions modéliser le jeu épistémique émergent qui serait visé par le professeur en référence au jeu épistémique source de la production d'une explication historique.

Dans un premier temps, pour tenter une réponse, les élèves s'en tiennent à une interprétation littérale du document, comme le montre la réponse de Cassandra (tdp 172). De même, Vincent s'en tient à une stricte définition des fonctions d'un personnage historique (tdp 170 : « Il dirige le peuple chrétien ») mais sa réponse ne permet pas de savoir s'il définit le pouvoir de Charlemagne ou celui du pape dont c'est effectivement la fonction. Un troisième élève, Elouan, remobilise un savoir antérieur déjà institutionnalisé dans la classe (tdp 174 à 176) pour proposer une interprétation de la scène du couronnement de Charlemagne. Il évoque la légendaire intervention de la colombe du "Saint-Esprit », lors du baptême de Clovis en 496, apportant la "Sainte-Ampoule » supposée contenir le "Saint-Chrême », cette huile « miraculeuse », dont les rois de France furent ensuite oints lors de leur sacre. Elle était supposée attribuer une validation divine au baptême de Clovis dont la conversion au christianisme lui assura le ralliement politique du clergé. La prégnance d'une signification contractuelle présente conduit l'élève à mobiliser une stratégie peu pertinente pour aborder une question nouvelle et produit à nouveau un envahissement du milieu par le contrat.

Le document iconographique joint représente cette croyance partagée durant tout le Moyen Âge. Il cherche à montrer que l'ampoule portée par la colombe vient directement du ciel et du Saint-Esprit.

Le baptême de Clovis par Rémi, évêque de Reims. Enluminure du XIVe siècle.

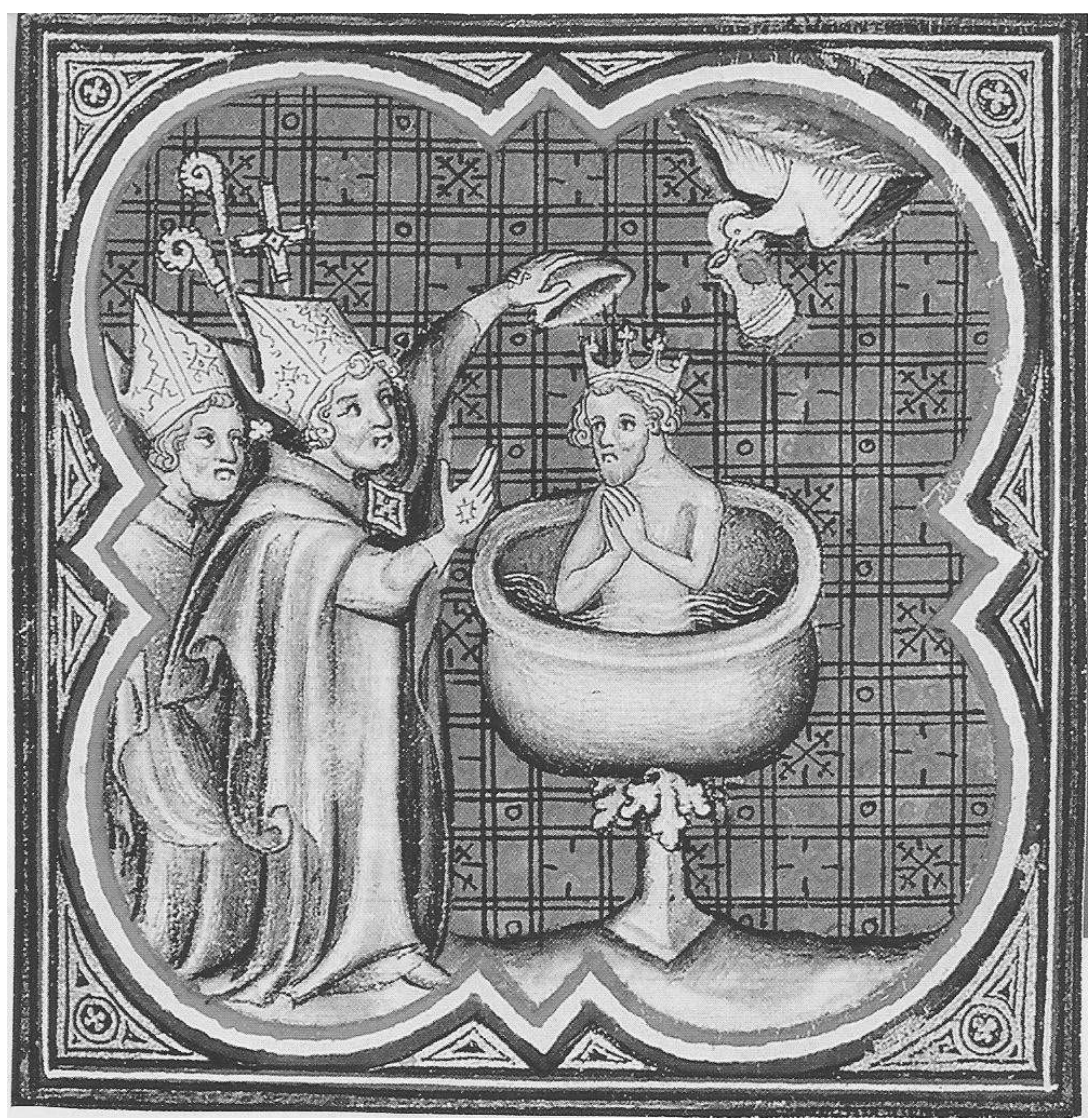

Manuel d'histoire et histoire des arts, classe de CM1, Paris : Nathan, 2010. 
Les interventions de ces trois élèves montrent à nouveau qu'ils procèdent à une lecture littérale du document afin d'en extraire des informations (par exemple: Charlemagne reçoit son pouvoir du pape) ou à la remobilisation d'un savoir antérieurement acquis (la colombe et l'ampoule) par une forme de jeu de devinette. Or, le professeur prévoyait que les élèves produiraient, à partir des informations fournies par le document, une interprétation supposant la mobilisation d'un savoir extérieur au document et nécessaire à l'explicitation de l'implicite du document. Comme le milieu n'est pas pourvoyeur des ressources nécessaires à cette explicitation, puisqu'il ne fournit aucune indication permettant cette inférence, le jeu glisse à nouveau et le professeur se voit obligé de fournir la réponse à sa propre question, par un effet Jourdain (tdp 177 : «Charlemagne reçoit les pouvoirs de Dieu »).

Les derniers échanges de cet extrait (Tissia, tdp 180 : «Parce que...»; Doriane, tdp 182: «Parce que... ») montrent que la compréhension d'une situation historique s'avère difficile pour des élèves hors d'une confrontation directe à un milieu pourvoyeur de significations. Le glissement du jeu d'apprentissage a conduit le professeur à fournir la réponse attendue aux élèves qui font ainsi faire l'économie d'une recherche d'hypothèses pour tenter d'expliquer l'action décrite sur le document. La seule explication du professeur ne leur permet pas de comprendre la signification de la scène représentée sur la miniature. À la différence de ses camarades pourtant, Vincent reprend à son compte l'explication $\mathrm{du}$ professeur et fournit des indications sur l'une des modalités de la compréhension historique. Il s'agit d'un mode de cognition naturelle et imageante qui spatialise les acteurs (Dieu en haut, le pape au milieu et l'empereur en bas) pour se représenter la nature de leur relation. Cette spatialisation a été suggérée par le professeur. Ce mode de cognition produit du sens par un transfert analogique (tdp 186 : le pouvoir transmis par le pape à l'empereur est « comme celui qui est entre Dieu et le pape) et par le recours à la compréhension narrative inscrite dans le schème de l'action (tdp 184 : « quand il se fait couronner »).

Cette courte séquence narrative produit une explication simplifiée du phénomène par le recours à un scénario logique (Sensevy \& Rivenc, 2003, p. 76-78 et p. 80-81). Un scénario logique est un scénario qui s'appuie sur la structure du récit (le 25 décembre 800 , le pape couronna Charlemagne) et il est logique en ce qu'il produit de la causalité historique afin de comprendre l'action décrite (le pape couronna Charlemagne afin de manifester l'origine divine de son pouvoir). Le scénario logique suppose la répétabilité des expériences humaines et des histoires du monde car, tout en n'étant jamais totalement identiques au fil du temps, ces expériences obéissent à des structures diachroniques au nombre limité et qui sont les manières d'agir stabilisées au sein des groupes humains (tdp 14 et 15 : la conquête militaire produit la constitution des empires, par exemple). Elles rendent l'histoire compréhensible car elles favorisent la comparaison entre des situations historiques structurellement proches et appartenant à une même série d'événements (Koselleck, 1997, p. 237 ; Lautier, 1997, p. 70-73). À cet égard, l'historien ne procède pas différemment des élèves : « Il transfère des modes d'explication qui ont fait leur preuve dans l'expérience sociale quotidienne de chacun » (Prost, 1996 : p. 159). Max Weber supposait que les pratiques des acteurs sociaux relevaient d'une typologie de conduites sociales facilement connaissables car elles se manifestent avec régularité dans une société donnée. Par exemple, une collision entre deux cyclistes relève d'un hasard malencontreux mais l'échange d'insultes qui risque de s'ensuivre est en revanche un comportement prédictible car régulier dans une telle situation (Weber, 1913, p. 321).

Un scénario logique relève de la compréhension narrative et de la cognition naturelle qui fondent l'apprentissage de l'histoire, car ces démarches rapprochent les savoirs nouveaux à acquérir de savoirs anciens déjà acquis afin de rendre compte d'une situation du passé (Cariou, 2012). Cependant, un scénario logique peut bloquer la compréhension quand un élève transfère un scénario déjà assimilé (par exemple, la colombe qui apporte la sainte ampoule lors du baptême de Clovis) vers une situation structurellement différente (le couronnement de Charlemagne). La difficulté ici, c'est que le milieu ne fournit ici aucune ressource pour invalider l'hypothèse de l'élève car rien dans l'image ne suggère aux élèves ce qui pourrait se trouver au-dessus du pape. Un hiatus apparait ici entre l'intention du professeur et les potentialités réelles du milieu. 


\section{Chercher les informations pertinentes sur une carte}

L'activité de prélèvement des informations dans un document pose également la question des critères $\mathrm{du}$ choix des informations pertinentes en fonction de l'intention du professeur. Nous assistons à nouveau à un envahissement du milieu par le contrat car l'interprétation du contrat par les élèves les pousse uniquement au prélèvement indifférencié d'informations sans se soucier de leur pertinence, comme l'indique l'extrait suivant qui intervient au début de la seconde séance, à l'occasion d'un retour sur la carte déjà analysée lors de la première séance.
Rivenc, 2003, p. 72-73). Cet extrait signale donc la nécessité d'outiller les élèves dans leur lecture de la carte afin de renouveler le contrat et de le rendre apte à explorer les potentialités du milieu. Il serait utile de faire porter l'attention des élèves sur les figurés cartographiques linéaires (les limites de l'empire), ponctuels (la localisation des grandes villes) et de surfaces (qui représentent des territoires). Ces figurés sont davantage pourvoyeurs de signification que le nom des royaumes eux-mêmes puisqu'ils permettent de distinguer les royaumes (figuré de surface) de la capitale (figuré ponctuel). Sans un renouvellement des stratégies contractuelles intégrant la reconnaissance des figurés cartographiques, il s'avère impossible de

\begin{tabular}{|l|l|l|}
\hline 408 & Prof & Est-ce que quelqu'un peut me citer les différents royaumes sur la carte ? Lila ? \\
\hline 409 & Lila & Royaume des Francs \\
\hline 410 & Prof & Alors, oui. \\
\hline 411 & Lila & Royaumes anglo-saxons \\
\hline 412 & Prof & Alors, est-ce que ça fait partie de l'empire ? \\
\hline 413 & Élèves & Non \\
\hline 414 & Prof & Non \\
\hline 415 & Lila & Aix-la-Chapelle... \\
\hline 416 & Prof & Aix-la-Chapelle, c'est quoi ? \\
\hline 417 & Élèves & C'est la capitale de Charlemagne \\
\hline 418 & Guillaume & Il y a la Saxe aussi \\
\hline$(\ldots)$ & & \\
\hline 425 & Élève & Rome \\
\hline
\end{tabular}

Les élèves ne travaillent pas dans le milieu car ils s'adonnent à un prélèvement indifférencié des informations sans se soucier de leur pertinence : les noms d'Aix-la-Chapelle et de Rome valent le nom des royaumes (tdp 415 et 425). Or, la lecture d'une carte historique suppose de nombreuses compétences déjà relevées par ailleurs : la reconnaissance de l'objet en tant que carte, la reconnaissance de l'espace représenté sur cette carte, l'identification du figuré et des informations portées sur cette carte (Sensevy \& dévoluer aux élèves la construction du savoir s'ils disposent seulement d'informations prélevées indifféremment dans le document.

\section{Lire un texte pour généraliser}

Lors de la lecture du texte à la fin de la seconde séance, ce qui est finalement dévolu aux élèves est la responsabilité d'une interprétation des mots du texte

\begin{tabular}{|l|l|l|}
\hline 541 & Prof & $\begin{array}{l}\text { Alors, à votre avis, quelles sont, en lisant le texte, là, on peut savoir quelles sont ses missions, au } \\
\text { comte ? Qu'est-ce que Charlemagne lui demande au comte ? }\end{array}$ \\
\hline$(\ldots)$ & & \\
\hline 550 & Vincent & Arrêter la violence \\
\hline 551 & Prof & Arrêter la violence. Dans notre pays, qui arrête la violence ? Quel pouvoir c'est ? Cassandra? \\
\hline 552 & Cassandra & Les policiers. \\
\hline 553 & Prof & Comment? \\
\hline 554 & Cassandra & Les policiers, ils arrêtent les crimes. \\
\hline 555 & Prof & Oui, les policiers. Là on s'écarte un petit peu. Moi j'attendais en fait la justice. \\
\hline
\end{tabular}


et le passage d'une information (par exemple : arrêter la violence) à une catégorie générale désignée par un hyperonyme (la justice) afin de mettre ensuite en évidence les différentes fonctions du comte.

Le milieu s'avère ici insuffisamment pourvoyeur de significations car rien, dans ce milieu, ne permet aux élèves de généraliser à partir des informations du texte. Les élèves ne sont pas outillés pour répondre aux intentions du professeur. Nous émettons donc l'hypothèse que la modélisation d'une situation d'apprentissage en terme de jeu d'apprentissage et l'appréhension des documents et des consignes en tant que milieu avec lequel le professeur met les élèves en situation d'interagir au moyen des stratégies présentes dans le contrat didactique, devrait permettre d'envisager le potentiel épistémique du milieu, en amont du déroulement de la séance. Contrairement à ce qui a été effectué lors de la préparation de la séance analysée ici, il serait utile d'évaluer cette capacité à actualiser des savoirs nouveaux sur le passé mais aussi à doter les élèves des pratiques raisonné, des savoirs nouveaux sur le passé (Moniot, 1993, p. 49-53). En conséquence, si nous admettons l'hypothèse d'une parenté entre le jeu épistémique source de critique de la source et le jeu épistémique émergent d'analyse du document en classe d'histoire, ce qui est susceptible de faire milieu, c'est ce qui conduirait les élèves à déployer des stratégies pour construire du savoir à partir de documents et non pour retrouver un savoir préexistant dans le document. Nous affirmons à nouveau la nécessité pour la communauté des didacticiens de l'histoire de mieux connaitre les pratiques historiennes et de les modéliser comme des jeux épistémiques sources afin d'en envisager une didactisation susceptible de rompre l'inertie du modèle disciplinaire.

Un peu plus loin pourtant, l'intention du professeur est de rassembler les informations prélevées dans le texte - conformément au modèle de conceptualisation dominant en histoire scolaire - afin de produire une définition du concept de comte en fonction de la consigne initiale du professeur (tdp 563).

\begin{tabular}{|l|l|l|}
\hline 563 & Prof & Si on voulait faire une définition sur le comte, quel mot on utiliserait ? (silence des élèves) \\
\hline 564 & Élève & On l'a déjà vu ? \\
\hline 565 & Prof & Un mot qu'on a déjà utilisé, oui. \\
\hline 566 & Élève & Justice \\
\hline 567 & Prof & Pourquoi tu parles de justice ? \\
\hline 568 & Élève & Parce que c'est lui qui arrête les coupables \\
\hline 569 & Prof & D'accord (silence des élèves). Cassandra? \\
\hline 570 & Cassandra & Président. \\
\hline 571 & Prof & Président? Un comte, c'est un président? \\
\hline
\end{tabular}

de savoir que l'on pourrait modéliser comme un jeu épistémique émergent. Privilégier ceux-là au détriment de celles-ci produit un rapport objectiviste aux documents laissant croire que le simple prélèvement d'informations permet la généralisation (Doussot, 2012). Nous rencontrons à nouveau une raison possible de l'inertie du modèle disciplinaire de l'histoire scolaire.

On oublie trop souvent que les traces du passé étaient destinées à parler à d'autres que nous et qu'elles n'ont pas été produites pour nous instruire sur le passé. Du point de vue de la pratique historienne, une trace n'est érigée en source historique que par les questions que lui adresse l'historien quand il considère qu'elle est susceptible de lui fournir, à la suite d'un traitement méthodique et
Le silence des élèves montre qu'ils ne parviennent pas à interpréter la consigne du professeur car, comme nous l'avons déjà vu, les stratégies contractuelles à leur disposition ne les poussent pas à conceptualiser pour tenter d'atteindre les significations du milieu. Elles les poussent à tenter un déchiffrement des intentions du professeur, à donner la priorité à des significations déjà-là, à retrouver des informations déjà institutionnalisées dans la classe (tdp 564 : « On l'a déjà vu ? »). Les élèves croient devoir jouer à un jeu de devinette qui les pousse à proposer au hasard des mots déjà évoqués durant ces trois séances mais sans lien avec l'activité en question (tdp 566: «Justice »; tdp 570 : « Président »).

L'analyse de cet extrait signale une possible confusion sur l'objet de savoir à enseigner et inter- 
roge la pertinence de faire chercher un hypéronyme aux élèves. Ces derniers ont bien perçu que le comte devait prélever les impôts et arrêter les bandits. Mais là ne réside pas la difficulté et l'intérêt du texte sur les comtes. D'autres stratégies ébauchées à d'autres moments de la séance semblent $a$ posteriori plus efficaces pour permettre une exploration des potentialités du milieu et un renouvellement du contrat didactique. L'essentiel tient en effet dans la relation de réciprocité qui unit le comte et Charlemagne, comme le signale le début du texte: le comte jure fidélité à Charlemagne («Ayant reconnu ta fidélité...») et Charlemagne confie sa charge au comte ( ....je te donne la fonction de comte de cette région »). En classe d'histoire, il est moins important de savoir quelque chose - de connaitre précisément chaque fonction du comte - que d'être capable de rendre compte des raisons qui expliquent ce fait : les raisons pour lesquelles Charlemagne institua les comtes et voulait s'assurer de leur fidélité. Cette relation de réciprocité permet de comprendre la fonction du comte : il gouverne - il chasse les bandits et il lève les impôts - le comté pour le compte de Charlemagne et il contribue ainsi à l'administration de ce vaste empire. La compréhension de cette phrase suppose la dévolution aux élèves de la production d'un scénario logique racontant les procédures de nomination du comte afin de verbaliser cette relation de réciprocité, comme cela a été tenté à un autre moment de la leçon que nous n'évoquerons pas ici.

L'institutionnalisation du scénario logique comme une stratégie contractuelle efficiente parait à nouveau susceptible de renouveler le contrat didactique pour que les élèves puissent travailler le milieu.

\section{DISCUSSION}

\section{Le modèle disciplinaire comme un effet de} contrat

Cette étude de cas met en lumière certaines des caractéristiques du contrat dominant en classe d'histoire qui pourraient expliquer les raisons de l'inertie du modèle disciplinaire réaliste de l'histoire enseignée. Précisons que les apories de ce contrat ne sont pas totalement imputables à l'inexpérience des étudiantes qui ont mené ces séances car un constat identique peut être fait au sujet de séances menées par des professeurs expérimentés (Tutiaux-Guillon, 2003 ; IGEN, 2005).

Nous avons montré que les élèves envisagent les documents comme "réalistes » ou « vrais » (Mia, tdp 154) car ils les perçoivent comme transparents à la réalité du passé. Leur nature importe peu et une carte historique peut être indifféremment un « document » ou une "fiche» (Guillaume, tdp 385-398). Une stratégie contractuelle inadéquate envahit à ce point le milieu qu'elle l'écrase et en gomme les spécificités. Ce qui compte, ce sont les informations fournies par ce document, si bien que la carte de l'empire de Charlemagne est identifiée par son objet même, à savoir l'empire ou les territoires conquis par Charlemagne (Guillaume et Alexandre, tdp 12 et 14). La seconde caractéristique du contrat renforce l'illusion de la transparence des documents en ce qu'elle pousse uniquement les élèves à y prélever des informations de manière indifférenciée, car toutes les informations se valent : pour désigner les royaumes de l'empire de Charlemagne, les élèves énoncent des noms de ville (Lila, tdp 415). En corollaire, le prélèvement d'informations conduit à tenter de reconnaitre un savoir déjà institué par un jeu de devinette (tdp 564 : «On l'a déjà vu ?»), comme le signale aussi l'évocation de la colombe et de l'ampoule (tdp 174-176).

Nous avons constaté en permanence l'envahissement du milieu par le contrat qui provoque le glissement du jeu d'apprentissage. Comme les stratégies contractuelles appellent uniquement le prélèvement ou la reconnaissance d'informations sous forme de devinette, il s'avère impossible de dévoluer aux élèves la responsabilité d'émettre des hypothèses interprétatives sur les faits étudiés. Le jeu didactique perd de sa densité épistémique et la régulation du jeu se voit réduite à une série d'effets de contrat, effets Topaze ou effets Jourdain.

Ces effets de contrat découlent d'une organisation du temps didactique par l'étude progressive d'une succession de documents isolés, par une succession de boucles didactiques caractéristiques des leçons d'histoire (Audigier et al., 1996) : le professeur introduit un document, il en fait préciser la nature par les élèves, il les questionne - oralement ou par écrit - sur le vocabulaire et les informations repérables dans le document et il reformule les réponses des élèves à des fins de généralisation, et ainsi de suite. Le milieu se constitue donc au fil de l'étude de documents successifs censés actualiser et redéfinir le 


\section{Didier Cariou}

savoir par empilement. Les caractéristiques de cette mésogénèse - par laquelle le chercheur rend compte de l'organisation des séances d'histoire et des caractéristiques du milieu - empêchent la confrontation des élèves au milieu car ils sont pris dans la course à la devinette. Elles produisent l'envahissement du milieu par le contrat ainsi qu'un glissement du jeu d'apprentissage qui découle de la nécessité de faire avancer le temps didactique. En effet, ce que l'on peut nommer la chronogénèse du savoir suppose qu'un certain nombre d'objets de savoir se succèdent tout au long de la leçon. Enfin, la place respective occupée par le professeur et les élèves dans leurs transactions autour du savoir, décrite en terme de topogenèse, signale la permanence d'une position topogénétique haute du professeur. Comme il ne peut simplement accompagner et réguler un jeu didactique qu'il aurait dévolu à ses élèves et qui aurait permis l'exploration du milieu par les élèves eux-mêmes, il est constamment obligé d'adopter une posture d'analyse, de validation ou d'invalidation des réponses des élèves et d'énonciation didactique qui laisse peu de place à l'initiative des élèves (Sensevy, 2011, p. 230). Ainsi, l'analyse en termes de triplet des genèses (Chevallard, 1991, p. 71-76 ; Schubauer-Leoni et al., 2007 ; Sensevy, 2011, p. 147-151) produit, elle aussi, une description des déséquilibres perçus dans l'analyse de ces séances d'histoire.

Le modèle disciplinaire de l'histoire enseignée n'est donc pas une contrainte qui s'impose fatalement aux séances d'histoire, il est le résultat de pratiques d'enseignements. Ces pratiques produisent une série de déséquilibres entre le contrat et le milieu entrainant des glissements du jeu d'apprentissage et réduisant la densité de l'apprentissage.

\section{Quelle équilibration possible entre le contrat et le milieu en classe d'histoire?}

Ces glissements posent la question du lien entre le jeu épistémique source et le jeu épistémique émergent pour modéliser les interactions en classe d'histoire. On pourrait considérer que ces effets de contrat viennent de l'écart finalement irréductible entre l'histoire scolaire et l'histoire savante et qu'ils tiennent à l'impossibilité de relier les jeux épistémiques émergents, qui modéliseraient la transmission d'une vulgate, aux jeux épistémiques source qui modélisent la construction du savoir savant.
Il semble toutefois que les nombreux malentendus analysés ici tiennent également à une caractéristique de l'historiographie elle-même. M. de Certeau (1987, p. 62-63) a montré que le récit historique était à la fois injonctif, car il parlerait au nom du réel pour le signifier, et performatif car, en signifiant efficacement le réel, il irait jusqu'à fabriquer du réel. Certes, les historiens ne sont pas dupes de leur propre discours et savent le mettre à distance, mais on peut se demander si la dimension réaliste du modèle disciplinaire de l'histoire enseignée ne découle pas de l'ambiguité du discours historien qui est discours scientifique sur le passé (histoire-science) et en même temps reconstruction du passé par des procédés d'écriture (histoire-art). Plus simplement, les difficultés mises en évidence ici pourraient tenir à la confusion entretenue entre le passé, qui est la réalité même, et l'histoire, qui est pratique de savoir et discours sur cette réalité passée. La dimension réaliste du modèle disciplinaire renverrait alors à l'épistémologie du savoir historique, aussi bien scolaire que savant.

Cette ambiguité est proche de celle qui préside à l'usage des documents en classe d'histoire puisque ces traces du passé sont perçues par les élèves comme le passé lui-même. Nous touchons ici à la question de l'usage des documents en classe d'histoire. Relativement absents des manuels et des classes jusque dans les années 1960, ils ont été introduits ensuite - sans réflexion approfondie - pour apporter $\mathrm{du}$ « concret » dans la classe d'histoire, afin de « motiver » les élèves et de les rendre " actifs» selon une logique de gestion de la classe, et pour les initier à la critique, dans une perspective civique. En conséquence, le document, originellement outil de l'historien et outil didactique, est mis au service de finalités générales qui ont fini par faire oublier sa fonction dans la construction du savoir et de l'apprentissage de l'histoire (Lautier, 1997, p. 175-177). Nous touchons à la complexité de l'épistémologie de la didactique d'une science indiciaire qui rend la question de l'usage des documents en classe d'histoire parente de celle des sources de l'histoire savante.

Nous émettons donc l'hypothèse qu'il conviendrait de revenir aux pratiques savantes des historiens - et notamment à leur pratique de critique des sources et d'inférence d'une généralité à partir des traces du passé érigées en sources par l'historien - et de les modéliser pour envisager les éléments qui pourraient en être effectivement didactisés et transposés dans la 
classe. Les analyses qui précèdent montrent que notre relative méconnaissance des pratiques effectives des historiens constitue un obstacle à nos investigations. Il semble donc impératif pour la communauté des didacticiens de l'histoire de poursuivre un travail d'élucidation déjà bien engagé (Doussot, 2011, 2012). Il s'agirait notamment de penser la fonction du document et sa capacité à « faire milieu » en classe d'histoire. Comme le montre la leçon analysée ici, l'enjeu serait de conduire les élèves à considérer les documents non comme le réel du passé mais comme des traces du passé questionnées par un problème et à partir desquelles ils pourraient construire du savoir sur le passé. Il s'agirait alors d'orienter le travail de la classe en la considérant comme un collectif instituant une version scolaire d'un collectif de savants (Sensevy, 2011, p. 128), comme une « communauté historienne scolaire » (Le Marec et al, 2009 ; Doussot, 2011). En effet, les élèves n'apprennent pas seulement l'histoire en construisant le savoir mais aussi en participant à une « communauté d'enquête » au sens pragmatiste du terme (Dewey, 1938).

Des recherches ultérieures devraient vérifier si le «nourrissement » du jeu d'apprentissage, grâce auquel on peut modéliser les pratiques d'apprentissage, par les jeux épistémiques sources par lesquels on peut modéliser les pratiques savantes des historiens (Sensevy, 2011, p. 741), pourrait devenir la condition de l'équilibration entre un contrat renouvelé et un milieu repensé et pourvoyeur de significations.

\section{CONCLUSION}

L'ensemble de cette analyse pointe la question du savoir mobilisé en classe d'histoire. La plupart du temps, le savoir apparait comme un objet en soi, essentialisé, il serait le résultat d'un jeu de devinette auquel on gagne si l'on a reconnu la bonne information dans un document (Sensevy, 2011, p. 122). Si l'on cherche au contraire à conduire les élèves à construire et s'approprier le savoir historique, nous émettons l'hypothèse que l'on devrait plutôt percevoir le savoir comme une "puissance d'agir » en situation au sein d'une institution (Sensevy, 2011). Cette puissance d'agir serait parente de celle que développent les communautés d'historiens. On concevrait alors le savoir comme une pratique qui rend capable d'agir en situation dans une communauté d'enquête et, en même temps, sur le plan épistémique, comme le résultat de cette pratique.

Cette conception praxéologique du savoir conduirait à envisager le savoir moins comme une connaissance en soi que comme une "assertion garantie », comme le produit d'une enquête bien menée - d'une "étude scientifiquement conduite", selon le mot de Lucien Febvre (1941) - à l'occasion de laquelle les élèves, sous la direction du professeur, mobilisent les processus d'hypothèses, de vérification et d'administration de la preuve au cœur de tout activité savante (Dewey, 1938, p. 65). Il s'avèrerait nécessaire de doter les élèves des outils qui leur permettraient de se confronter au milieu pour vérifier en pratique la pertinence d'une hypothèse ou d'un scénario logique, afin de les rendre capables des déplacements supposés par le modèle d'apprentissage de l'histoire. En d'autres termes, il s'agirait de construire un contrat didactique adéquat au milieu en ce qu'il doterait les élèves des systèmes de règles définitoires et de règles stratégiques qui leur permettaient de comprendre « à quoi l'on joue » en classe d'histoire afin d'y « faire ce qu'il y a à faire ». 


\section{RÉFÉRENCES}

Audigier F. (1995). Histoire et géographie : des savoirs scolaires en question entre les définitions officielles et les constructions des élèves. Spirales. Revue de recherche en éducation, $\mathrm{n}^{\circ} 15$, p. 61-89.

Audigier F. (2005). Les enseignements d'histoire et de géographie aux prises avec la forme scolaire. Maulini O. \& Montandon C. (dir.). Les formes de l'éducation : variétés et variations. Bruxelles: De Boeck Université, p. 103122.

Audigier F., Crémieux C. \& Mousseau M.-J. (1996). L'enseignement de l'histoire et de la géographie en troisième et en seconde. Etude descriptive et comparative. Paris : INRP.

Audigier F. \& Tutiaux-Guillon N. (dir.) (2004). Regards sur l'histoire, la géographie et l'éducation civique à l'école élémentaire. Saint-Fons : INRP.

Bloch M. (1949/1993). Apologie pour l'histoire ou Métier d'historien. Paris : Armand Colin.

Brousseau G. (1998). Théorie des situations didactiques. Grenoble : La pensée sauvage.

Cariou D. (2012). Écrire l'histoire scolaire. Quand les élèves écrivent en classe pour apprendre l'histoire. Rennes : PUR, collection Paideia.

Certeau M. de (1987/2002). Histoire et psychanalyse. Entre science et fiction. Paris : Gallimard, Folio.

Chervel A. (1998). La culture scolaire. Une approche historique. Paris: Belin.

Chevallard Y. (1991), La transposition didactique. Du savoir savant au savoir enseigné. $2^{\mathrm{e}}$ édition. Grenoble: La pensée sauvage.

Dewey J. (1938/1967). Logique: la théorie de l'enquête. Trad. fr., Paris : PUF.

Doussot S. (2010). Pratiques de savoir en classe et chez les historiens : une étude de cas au collège. Revue française de pédagogie $\mathrm{n}^{\circ} 173$, p. 85-104.

Doussot S. (2011). Didactique de l'histoire. Outils et pratiques de l'enquête historienne en classe. Rennes: PUR, collection Paideia.

Doussot S. (2012). Le cas Menocchio et la construction en histoire. Une lecture didactique de l'étude de cas selon Carlo Ginzburg. Le cartable de Clio n ${ }^{\circ} 12$ : p. 111-125.

Febvre L. (1941/1995). Vivre l'histoire. Propos d'initiation. Combats pour l'histoire. Paris : Pocket Agora, p. 18-33.

Ginzburg C. (1986/1989). Mythes, emblèmes, traces. Morphologie et histoire. Trad. fr. Paris : Flammarion.

IGEN (2005). Sciences expérimentales et technologiques, histoire et géographie. Leur enseignement au cycle III de l'école primaire. Rapport $\mathrm{n}^{\circ}$ 2005-112.

http://www.education.gouv.fr/cid2195/sciences-experimentales-et-technologie-histoire-et-geographie-leurenseignement-au-cycle-iii-de-l-ecole-primaire.html (consulté le 2 octobre 2012).

Koselleck R. (1997). L'expérience de l'histoire. Trad. fr. Paris : Gallimard / Le Seuil.

Kuhn T. S. (1977/1990). La tension essentielle. Tradition et changement dans les sciences. Trad. fr. Paris : Gallimard.
Lautier N. (1997). A la rencontre de l'histoire. Villeneuve d'Ascq : Presses du Septentrion.

Le Marec Y., Doussot S. \& Vezier A. (2009). Savoirs, problèmes et pratiques langagières en histoire. Éducation et didactique, vol. 3, n 3, p. 7-27.

Moniot H. (1993). Didactique de l'histoire. Paris, Nathan.

Lefeuvre L. (2012). Travail épistémique du professeur et transposition des savoirs. Gruson B., Forest D. \& Loquet M. (dir.). Jeux de savoir. Étude de l'action conjointe en didactique. Rennes: PUR, p. 335-352.

Marlot C \& Toullec-Théry M. (2011). Caractérisation didactique des gestes ordinaires à l'école élémentaire : une étude comparative de deux cas didactiques limite en mathématiques. Éducation et didactique, vol. 5, $\mathrm{n}^{\circ} 3$, p. 7-32.

Passeron J.-Cl. \& Revel J. (dir.) (2005). Penser par cas. Paris : Éditions de l'EHESS.

Prost A. (1996). Douze leçons sur l'histoire. Paris : Le Seuil, Points.

Riché P. (1983/2012). Les Carolingiens. Une famille qui fit l'Europe. Paris : Hachette, rééd. Pluriel.

Schubauer-Leoni M.-L., Leutenegger F., Ligozat F. \& Flückiger A. (2007). Un modèle de l'action conjointe professeur-élèves : les phénomènes didactiques qui peut/ doit traiter. Sensevy G. \& Mercier A. (dir.). Agir ensemble. Laction didactique conjointe du professeur et des élèves. Rennes : PUR, p. 51-91

Sensevy G. (2011). Le sens du savoir. Éléments pour une théorie de l'action conjointe en didactique. Bruxelles: De Boeck.

Sensevy G. \& Rivenc J.-P. (2003). Un enseignement de l'histoire au CM. Questions didactiques. Revue Française de Pédagogie $\mathrm{n}^{\circ} 144$, p. 69-83.

Tutiaux-Guillon N. (2003). Lhistoire scolaire en France. Un modèle résistant aux débats et aux controverses. Baquès M.-C., Bruter A. Tutiaux-Guillon N. (dir.). Pistes didactiques et chemins d'historiens. Textes offerts à Henri Moniot. Paris : L'Harmattan, p. 267-284.

Tutiaux-Guillon N. (2009). L'histoire scolaire française entre deux modèles: contenus, pratiques et finalités. Revue Française d'éducation comparée $\mathrm{n}^{\circ} 4$ : p. 105-116.

Tutiaux-Guillon N. \& Mousseau M.-J. (1998). Dans la classe : l'influence du modèle pédagogique sur l'explicatif. Audigier F. (dir). Contributions à l'étude de la causalité et des productions des élèves dans l'enseignement de l'histoire et de la géographie. Paris, INRP : p. 33-68.

Verret M. (1975). Le temps des études. Paris : Honoré Champion.

Weber M. (1913/1992). Essai sur quelques catégories de la sociologie compréhensive. Essais sur la théorie de la science. Trad.fr. Paris : Pocket Agora, p. 301-364. 